\title{
多组分反应合成紫杉醇侧链及其在合成紫杉醇衍生物中的应用
}

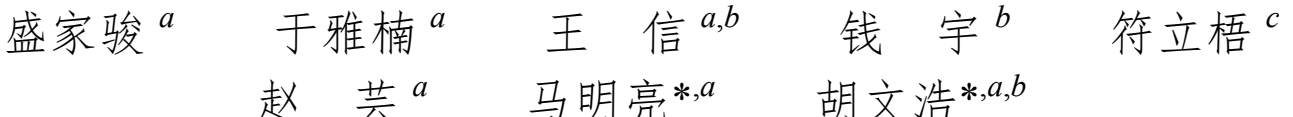 \\ ( ${ }^{a}$ 华东师范大学上海分子治疗与新药创制工程技术研究中心 上海 200062) \\ ${ }^{b}$ 中山大学药学院 广州 510006) \\ ( ${ }^{c}$ 中山大学肿瘤防治中心 广州 510060)
}

\begin{abstract}
摘要 以水、重氮乙酸酯、芳香醛和芳香胺为原料, 在单一催化剂 $\mathrm{Cu}(\mathrm{OTf})_{2}$ 的作用下成功实现了一例基于羟基叶立德 捕捉的四组分反应，一步构建了 $\alpha$-羟基- $\beta$-氨基酸酯类化合物骨架，并通过进一步的化学转化高效地合成了紫杉醇朢唑 烷型侧链. 此外, 将所得侧链与紫杉醇母核、卡巴他赛母核对接, 成功合成了 14 个紫杉醇衍生物, 并在随后的抗肿瘤 细胞活性测试中, 发现了多个活性优异的新型化合物. 将多组分反应方法学与应用相结合, 开发了一种高效合成紫杉 醇侧链衍生物的方法, 并成功地将其应用到紫杉醇衍生物的半合成中, 为该类化合物的构效关系研究提供了一种新途 径, 具有潜在的应用价值.

关键词 紫杉醇侧链; 紫杉醇衍生物; 多组分反应; 羊基叶立德
\end{abstract}

\section{Synthesis of Paclitaxel Side Chain via Multi-Component Reaction and Its Application to the Synthesis of Paclitaxel Analogues}

\author{
Sheng, Jiajun ${ }^{a}$ \\ Yu, Ya'nan ${ }^{a}$ \\ Wang, Xin ${ }^{a, b}$ \\ Qian, $\mathrm{Yu}^{b}$ \\ $\mathrm{Fu}, \mathrm{Liwu}^{c}$ \\ Ma, Mingliang*,a $\mathrm{Hu}$, Wenhao ${ }^{*, a, b}$ \\ ( ${ }^{a}$ Shanghai Engineering Research Center of Molecular Therapeutics and New Drug Development, \\ East China Normal University, Shanghai 200062) \\ $\left({ }^{b}\right.$ School of Pharmaceutical Sciences, Sun Yat-sen University, Guangzhou 510006) \\ ( ${ }^{c}$ Cancer Center, Sun Yat-sen University, Guangzhou 510060)
}

Zhao, $\operatorname{Yun}^{a}$

\begin{abstract}
Under the catalysis of $\mathrm{Cu}(\mathrm{OTf})_{2}$, a hydroxy ylide trapping based multi-component reaction using water, diazoacetates, aromatic aldehydes and aromatic amines was successfully carried out to construct $\alpha$-hydroxy- $\beta$-amino acid ester skeleton in one step. The taxol oxazolidine-type side chains were efficiently synthesized after further chemical transformations. Moreover, 14 taxane derivatives were synthesized through esterification of obtained side chains and 7-O-(triethylsilyl) baccatin III or 7,10-dimethoxy-10-beacetyl baccatin III, and several novel compounds with excellent activity were discovered in the test of anti-tumor activity. In this paper, combining the methodology and application of multi-component reaction, a highly efficient method for synthesizing paclitaxel side chain derivatives was developed, which was applied into the semi-synthesis of paclitaxel analogues. This research provided a new approach to the study of structure-activity relationship and had potential application value.
\end{abstract}

Keywords taxol side chain; paclitaxel analogues; multi-component reaction; hydroxy ylide

紫杉醇(paclitaxel, 商品名 $\mathrm{Taxol}^{\circledR}$ ) 是从太平洋红杉 的树皮中提取出来的一种具有抗肿瘤活性二萜类天然 产物, 它与其结构改造衍生物多西紫杉醇(docetaxel)和 卡巴他赛(cabazitaxel)均是迄今为止发现最有效的广谱
抗肿瘤药物之一 ${ }^{[1]}$. 目前主要用于治疗乳腺癌、卵巢癌 等, 还可与顺铂、吉西他滨、阿霉素、曲妥珠单抗等联 合用药, 用于治疗非小细胞肺癌、转移性乳腺癌、AIDS (Acquired Immune Deficiency Syndrom)相关的卡式肉瘤

* Corresponding authors. E-mail: mlma@brain.ecnu.edu.cn; huwh9@mail.sysu.edu.cn Received July 24, 2018; revised December 17, 2018; published online December 21, 2018. Project supported by the National Natural Science Foundation of China (Nos. 21572067, 21332003). 国家自然科学基金(Nos. 21572067, 21332003)资助项目. 
等多种癌症 ${ }^{[2]}$. 然而, 紫杉醇在红豆杉树皮中的含量极 低，仅有 $0.069 \%$ ，从树皮中分离提取不仅无法满足用药 需求，还会对红豆杉造成致命的损伤，导致其死亡 ${ }^{[3]}$. 此外, 紫杉醇的化学结构极其复杂, 化学全合成仅停留 在实验室水平 ${ }^{[4]}$, 因此目前生产紫杉醇的主要方法是由 10-去乙酰基巴卡汀 III (10-DAB)为原料的半合成法 (Scheme 1) ${ }^{[5]}$. 紫杉醇 C-13 侧链是半合成路线中至关重 要的一个片断, 为了寻找一条便捷高效的合成路线, 多 年来全世界各国化学家都付诸了许多努力. 该侧链属于 $\alpha$-差基- $\beta$-氨基酸酯类化合物，对于这一类化合物， Sharpless 不对称着胺化 ${ }^{[6]}$ 、Sharpless 不对称羟基化 ${ }^{[7]}$ 、 环氧或氮杂环丙烷的选择性开环等方法 ${ }^{[8]}$ 都曾被报道应 用于它们的合成中. 除此之外, 过渡金属催化的多组分 反应包括 Mannich 型 ${ }^{[9]}$ 和 1,3-偶极环加成型 ${ }^{[10]}$, 也都是 合成紫杉醇侧链的有效方法. 我们课题组 ${ }^{[9 a}$ 曾在 2010 年报道了一例由醋酸铑与手性磷酸共同催化的三组分 反应合成紫杉醇侧链的方法, 并在此基础上, 于 2018 年 ${ }^{[10 c]}$ 发现了一种通过羰基叶立德多组分 $[3+2]$ 环加成 反应一锅法合成紫杉醇侧链的方法. 但这两类方法有一 个共同的特点, 它们都需要使用手性配体或昂贵的过渡 金属催化剂, 这直接导致该类反应在应用上受到了很大 的限制. 因此, 我们试图寻找一种廉价高效的催化体系 来催化该类多组分反应, 并探索使用水这样一种绿色原 料代替醇类作为羟基叶立德来源, 在提高原子经济性的 同时减少工艺步骤，使该方法具有一定的应用前景.

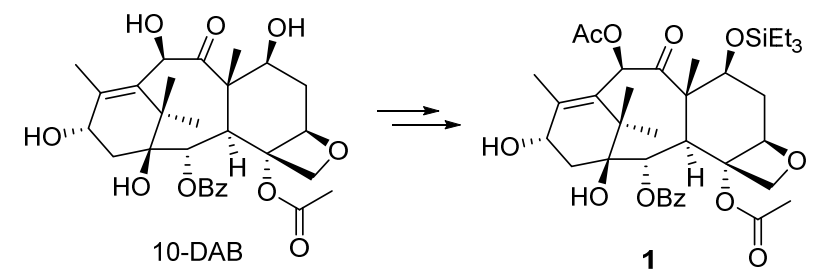<smiles>CO[C@@H]1C(=O)N(C(=O)c2ccccc2)[C@@H]1c1ccccc1</smiles><smiles>O=C(O)C1OC([18O])N(C(=O)c2ccccc2)C1c1ccccc1</smiles>

$\beta$-lactam type

\section{1 结果与讨论}

循着以上思路，我们首先对金属催化剂进行了研 究, 篮选了价格低廉且催化效果较为优秀的 $\mathrm{Cu}$ 催化剂 和 $\mathrm{Fe}$ 催化剂(表 1). 同时, 根据课题组以往的经验, 我们 用芳香氨 $3 a$ 和苯甲醛 $(4 a)$ 原位制备反应所需亚胺，进一 步减少反应步骤.

表 1 金属催化剂的篮选 ${ }^{a}$

Table 1 Selection of metal catalysis

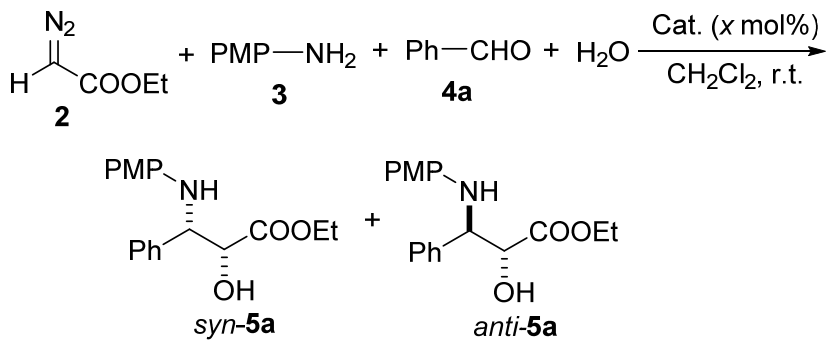

\begin{tabular}{clccc}
\hline Entry & \multicolumn{1}{c}{ Catalyst } & $x / \mathrm{mol}^{2} \%$ & $\mathrm{Yield}^{b} / \% d r^{c}($ syn $:$ anti $)$ \\
\hline 1 & $\mathrm{Rh}_{2}(\mathrm{OAc})_{4}$ & 2 & 62 & $58: 42$ \\
2 & $\mathrm{Cu}(\mathrm{OTf})_{2}$ & 10.0 & 57 & $44: 56$ \\
3 & $\mathrm{Cu}(\mathrm{OTf})_{2}$ & 5.0 & 58 & $45: 55$ \\
4 & $\mathrm{Cu}(\mathrm{OTf})_{2}$ & 2.0 & 52 & $45: 55$ \\
5 & $\mathrm{Cu}(\mathrm{OTf})_{2}+p-\mathrm{TsOH}$ & 5.0, & 60 & $38: 62$ \\
& & 10.0 & & \\
6 & $\mathrm{Cu}(\mathrm{OTf})_{2}+\mathrm{AgPF}_{6}$ & 5.0, & 46 & $52: 48$ \\
7 & $\mathrm{Cu}\left(\mathrm{CH}{ }_{3} \mathrm{CN}\right)_{4} \mathrm{BF}_{4}$ & 5.0 & 43 & $41: 59$ \\
8 & $\mathrm{Cu}(\mathrm{AcAc})_{2}$ & 5.0 & - & - \\
9 & $\mathrm{CuSO}$ & 5.0 & - & - \\
10 & $\mathrm{FeCl}$ & 5.0 & - & - \\
11 & $\mathrm{Fe}(\mathrm{OTf})_{3}$ & 5.0 & - & - \\
\hline
\end{tabular}

${ }^{a}$ The reaction was carried out with aniline 3a (1 equiv.), aldehyde 4a (1.05 equiv.), $\mathrm{H}_{2} \mathrm{O}$ (3 equiv.) and catalyst $(2.0 \sim 20.0 \mathrm{~mol} \%)$ in $\mathrm{CH}_{2} \mathrm{Cl}_{2}$ at room temperature with addition of EDA 2 ( 1.5 equiv.) over $1 \mathrm{~h} .{ }^{b}$ Isolated yield (two diastereoisomers). ${ }^{c}$ Determined by ${ }^{1} \mathrm{H}$ NMR spectroscopy of the crude reaction mixture.

如表 1 所示，使用 $5 \mathrm{~mol} \%$ 的 $\mathrm{Cu}(\mathrm{OTf})_{2}$ 时可以得到 更好的结果，对甲苯磺酸 $(p-\mathrm{TsOH})$ 共催化可以提升反应 收率但是会显著降低反应的非对映选择性, $\mathrm{AgPF}_{6}$ 共催 化虽然可以小幅提升非对映选择性，但是会降低收 率 ${ }^{[11]}$. 其余的铜催化剂中只有 $\mathrm{Cu}\left(\mathrm{CH}_{3} \mathrm{CN}\right)_{4} \mathrm{BF}_{4}$ 能够成 功催化该多组分反应, 但是结果不尽人意. 除此之外, 我们还篮选了一些其他廉价易得的 $\mathrm{Fe}$ 催化剂, 但是它 们都无法使目标多组分反应发生.

经过上述实验，虽然在 $\mathrm{Cu}(\mathrm{OTf})_{2}$ 催化下我们得到的 顺式构型(syn)的比例相对使用 $\mathrm{Rh}_{2}(\mathrm{OAc})_{4}$ 时有小幅减 少, 但是 $\mathrm{Cu}(\mathrm{OTf})_{2}$ 低廉的售价及较好的催化活性使它在 放大实验中仍然具有优势，因此我们仍然决定使用 $\mathrm{Cu}(\mathrm{OTf})_{2}$ 作为该路线的催化剂.

随后，进一步篮选了反应溶剂和反应温度(表 2). 结 
果显示, 使用二氯甲烷作溶剂时, 无论升温或降温都会 降低反应的收率; 而二氯甲烷以外的其他非质子型溶剂 都会在一定程度上降低反应收率或非对映选择性. 反应 条件篮选完毕后, 将反应规模由 $50 \mathrm{mg}$ 提升至 $10 \mathrm{~g}$ (表 2, Entry 9), 得到的结果与表 1 Entry 3 中的数据基本吻 合，这也可以证明该反应体系稳定，具有放大的前景.

表 2 溶剂与温度的篮选

Table 2 Optimization of solvent and temperature ${ }^{a}$

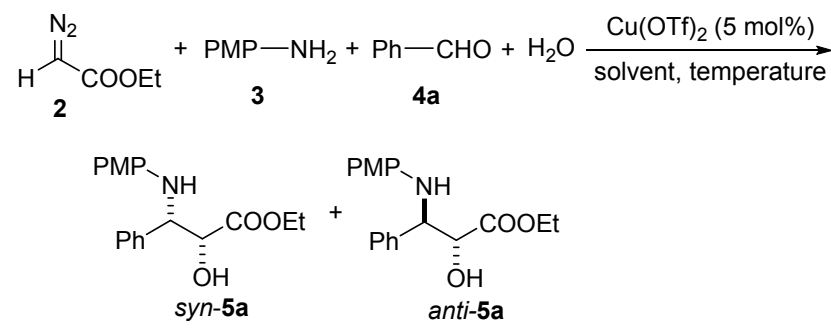

\begin{tabular}{clccc}
\hline Entry & Solvent & Temp. $/{ }^{\circ} \mathrm{C}$ & Yield $^{b} / \%$ & $d r^{c}(s y n:$ anti $)$ \\
\hline 1 & $\mathrm{CH}_{2} \mathrm{Cl}_{2}$ & 0 & 53 & $45: 55$ \\
2 & $\mathrm{CH}_{2} \mathrm{Cl}_{2}$ & Reflux & 47 & $43: 57$ \\
3 & $\mathrm{THF}$ & 25 & $<10$ & - \\
4 & $\mathrm{CHCl}_{3}$ & 25 & 54 & $42: 58$ \\
5 & $\mathrm{CH}_{3} \mathrm{CN}$ & 25 & - & - \\
6 & EtOAc & 25 & 45 & $37: 63$ \\
7 & Hexane & 25 & 34 & $46: 54$ \\
8 & Toluene & 25 & 30 & $44: 56$ \\
$9^{d}$ & $\mathrm{CH}_{2} \mathrm{Cl}_{2}$ & 25 & 56 & $45: 55$ \\
\hline
\end{tabular}

${ }^{a}$ The reaction was carried out with aniline 3a (1 equiv.), aldehyde 4a (1.05 equiv.), $\mathrm{H}_{2} \mathrm{O}$ (3 equiv.) and $\mathrm{Cu}(\mathrm{OTf})_{2}(5.0 \mathrm{~mol} \%)$ in the solvent with addition of EDA 2 (1.5 equiv.) over $1 \mathrm{~h} .{ }^{b}$ Isolated yield (two diastereoisomers). ${ }^{c}$ Determined by ${ }^{1} \mathrm{H}$ NMR spectroscopy of the crude reaction mixture. ${ }^{d}$ The reaction was carried out on $10 \mathrm{~g}$ scale.

脱除对甲氧基苯基(PMP)保护也是反应路线中相当 重要的一步. 在该步反应中, 我们选择使用一种温和的 氧化剂三氯异氧腿酸(TCCA) 以替代通常使用的硝酸铈 铵(CAN), 这样不仅避免了 $\mathrm{OH}$ 的保护, 还简化了后处 理步骤, 从而大大提高了反应收率 ${ }^{[12]}$. 最终通过重结晶 以 $60 \%$ 的收率得到化合物 $\mathbf{6 a}$, 该步反应同样可顺利放 大至 10 克级(Scheme 2). 之后, 将所得到的氨基醇 6a 在 乙酸乙酯和饱和碳酸氢钠水溶液的两相体系中进行苯 甲酰化, 所得粗产物经过萃取、分液后再经乙酸乙酯石油醚体系重结晶, 得到高纯度的白色固体 7a. 化合物 $7 \mathrm{a}$ 在有分水器存在的条件下与对甲氧基苯甲醛二甲缩 醛进行环化, 得到化合物 8a ${ }^{[13]}$. 最后将化合物 8a 进行 碱性水解, 得到目标噁唑烷型侧链 9a, 三步反应的总收 率可以达到 $67 \%$.

纵观整条合成路线, 每一步反应均成功地放大至 10 克级, 且操作步骤不需要改变, 得到的结果也与小试 时几乎一致. 在纯化产物时, 只有在分离非对映异构体 syn-5a、anti-5a 和分离油状液体 $8 \mathbf{a}$ 时使用了柱层析
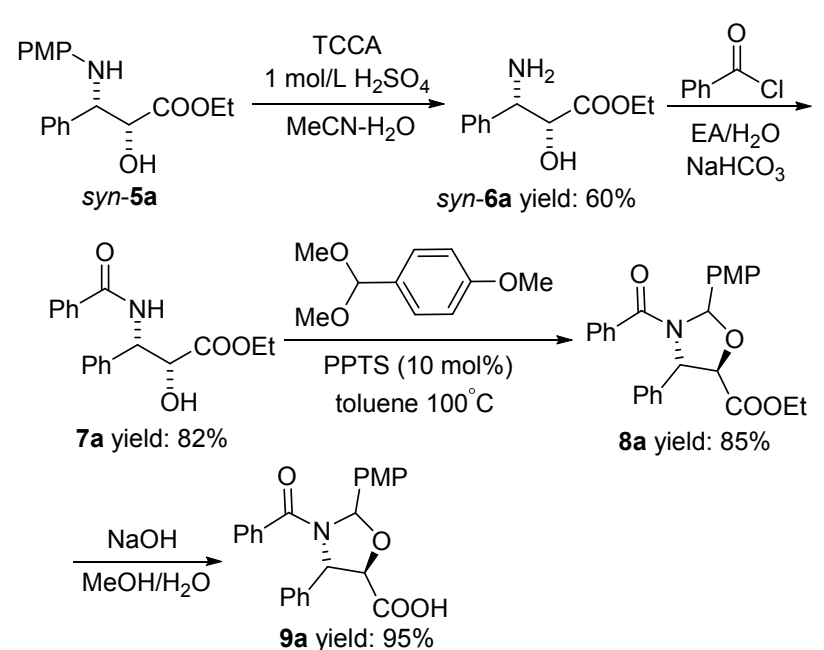

图式 2 噁唑烷型侧链合成路线

Scheme 2 Synthesis route of oxazoline type side chain

分离, 其余步骤通过简单的重结晶或过滤就可以得到较 高纯度的产物. 以苯胺为起始原料, 以顺式噁唑烷型侧 链 9a 为终产物计, 5 步反应的总收率为 $10 \%$, 相较先前 报道的路线有了很大的提高.

之后我们选用不同的芳香醛和酰氯对底物进行了 拓展. 令人惊喜的是, 各步反应均展现出了良好的底物 适应性. 最终得到了 14 种不同的紫杉醇噁唑烷型 C-13 侧链(Scheme 3).

在此基础上，我们成功地将 7-三乙基硅基巴卡汀 III (10, 7-TES Baccatin III) 和 7,10-二甲氧基-10-去乙酰 基巴卡汀 III (11, 7,10-dimethoxy-10-DAB)与前述所得的 噁唑烷型侧链进行缩合得到紫杉醇衍生物前体 12 或 13 , 最后在无水乙醇中进行酸解脱保护，得到了 14 种紫杉 醇衍生物(Scheme 4). 随后我们对几种代表性的肿瘤细 胞进行了活性测试(表 3). 结果显示, 对于所检测的 4 种 肿瘤细胞, 有多种紫杉醇衍生物的活性优于 paclitaxel 或 cabazitaxel. 其中化合物 14k 和 $15 \mathrm{~h}$ 表现最为优秀, 对 于 4460 大细胞肺癌和 PC9 肺腺细胞癌细胞的活性要优 于 paclitaxel, 对于 MG63 骨肉瘤和 KB 口腔表样癌细胞 的活性要同时优于 paclitaxel 和 cabazitaxel. 这一结果反 映了紫杉醇 7,10-位的甲基化取代和侧链 3'-位酰胺的杂 环取代会对活性产生非常积极的影响，而 3'-位磺酰胺 取代会大大降低化合物对肿瘤细胞的活性.

\section{2 结论}

研究了通过重氮乙酸乙酯(EDA)、水、芳香胺和芳 香醛的四组分反应合成紫杉醇 C-13 侧链前体的方法. 从经济性和实用性出发，篮选了一系列金属催化剂，最 终确定使用廉价、催化活性又相对较好的 $\mathrm{Cu}(\mathrm{OTf})_{2}$ 替代 $\mathrm{Rh}_{2}(\mathrm{OAc})_{4}$. 在后续多组分产物到噁唑烷型侧链的合成 中, 将原先所需的 6 到 7 步反应缩减为 4 步, 简化了后 

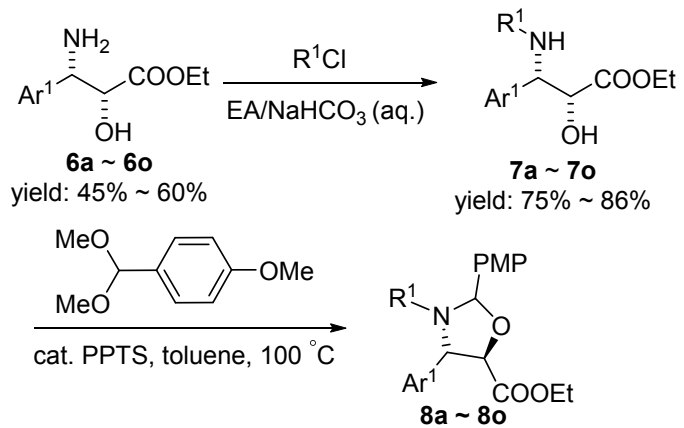

yield: $73 \% \sim 85 \%$

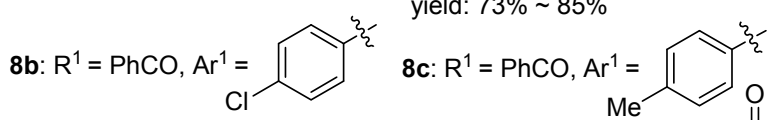

8d: $\mathrm{R}^{1}=\mathrm{PhCO}, \mathrm{Ar}^{1}=$

8f: $\mathrm{Ar}^{1}=\mathrm{Ph}, \mathrm{R}^{1}=$<smiles>[R17]C(F)(F)CC=C</smiles>

8j: $\mathrm{Ar}^{1}=\mathrm{Ph}, \mathrm{R}^{1}=$

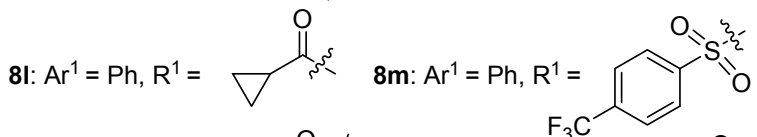

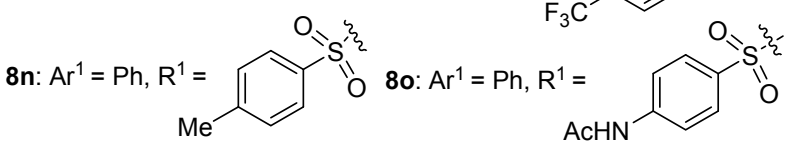

图式 3 底物拓展

Scheme 3 Scope extension of the substrate

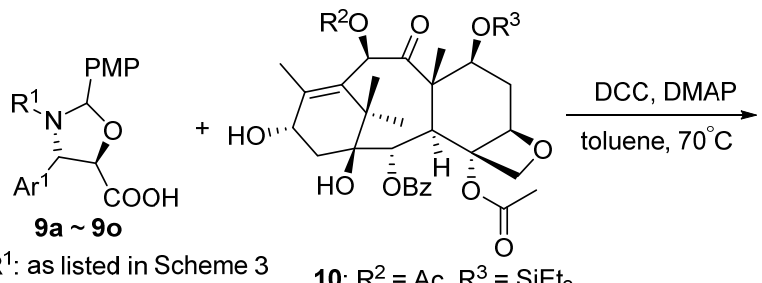

$\mathrm{R}^{1}$ : as listed in Scheme 3

$\mathrm{Ar}^{1}$ : as listed in Scheme 3

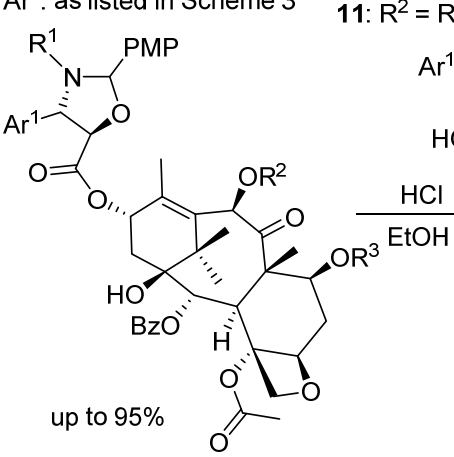

12: $R^{2}=A c, R^{3}=\mathrm{SiEt}_{3}$

13: $R^{2}=R^{3}=M e$

图式 4 紫杉醇衍生物的合成

Scheme 4 Synthesis of the paclitaxel analogues
表 3 紫杉醇衍生物的抗肿瘤细胞活性 ${ }^{a}$

Table 3 Anti-tumor activity of the paclitaxel analogues

\begin{tabular}{lccccc}
\hline \multirow{2}{*}{ Entry } & \multirow{2}{*}{$M_{\mathrm{r}}$} & \multicolumn{4}{c}{$\mathrm{IC}_{50} /\left(\mu \mathrm{mol} \cdot \mathrm{L}^{-1}\right)$} \\
\cline { 3 - 6 } & & $\mathrm{H} 460$ & $\mathrm{MG63}$ & $\mathrm{KB}$ & $\mathrm{PC} 9$ \\
\hline Paclitaxel & 853.33 & 0.0119 & 0.0115 & 0.0006 & 0.0031 \\
anti-14b & 888.36 & $>50$ & $>50$ & 9.6593 & 30.6455 \\
14e & 883.34 & 0.0203 & 0.0218 & $\mathbf{0 . 0 0 0 4}$ & 0.0064 \\
$\mathbf{1 4 h}$ & 896.37 & 0.0152 & 0.0455 & 0.0022 & 0.0145 \\
$\mathbf{1 4 i}$ & 913.35 & 0.1551 & 0.2918 & 0.0594 & 0.0930 \\
$\mathbf{1 4 j}$ & 843.31 & $\mathbf{0 . 0 0 4 6}$ & $\mathbf{0 . 0 0 5 9}$ & $\mathbf{0 . 0 0 0 5}$ & 0.0046 \\
$\mathbf{1 4 k}$ & 859.29 & $\mathbf{0 . 0 0 3 7}$ & $\mathbf{0 . 0 0 1 4}$ & $<\mathbf{0 . 0 0 0 5}$ & $\mathbf{0 . 0 0 1 0}$ \\
$\mathbf{1 4 m}$ & 957.29 & 13.7922 & 20.3900 & 6.0461 & 13.7886 \\
Cyclo-14m & 1075.33 & $>50$ & $>50$ & $>50$ & $>50$ \\
Cyclo-14n & 1021.36 & $>50$ & $>50$ & 30.4081 & $>50$ \\
Cabazitaxel & 835.38 & $<0.0005$ & 0.0080 & $<0.0005$ & $<0.0005$ \\
15a & 839.35 & 0.0179 & $\mathbf{0 . 0 0 2 9}$ & $<\mathbf{0 . 0 0 0 5}$ & $\mathbf{0 . 0 0 1 2}$ \\
15h & 882.39 & $\mathbf{0 . 0 0 1 8}$ & $\mathbf{0 . 0 0 4 6}$ & $<\mathbf{0 . 0 0 0 5}$ & $\mathbf{0 . 0 0 1 7}$ \\
\hline
\end{tabular}

${ }^{a}$ The data provided by collaborators from School of Pharmaceutical Sciences, Sun Yat-sen University.

处理步骤，最终以 $10 \%$ 左右的总收率得到了共 14 种顺 式噁唑烷型紫杉醇侧链. 该路线中每一步反应均可放大 至 $10 \mathrm{~g}$, 且操作步骤不需要改变, 得到的结果也与小试 时几乎一致，拥有一定的应用价值. 随后利用所得侧链， 合成了 14 种紫杉醇衍生物, 并在进一步的抗肿瘤细胞 活性测试中发现了多个活性优异的新型化合物.

值得注意的是, 我们成功地将多组分方法学的研究 成果应用到了紫杉醇类似物的合成中，大大提高了侧链 的多样性和合成效率，为后续的构效关系研究提供了相 当大的便利. 这也是多组分反应方法学应用价值的体 现.

\section{3 实验部分}

\section{1 仪器与试剂}

核磁共振氢谱和碳谱由 Bruker Avance III-400 核磁 共振仪测定; 高分辨质谱由 HRMS (ESI) Waters Synapt 质谱仪测定；高效液相色谱(HPLC)由 Thermol Scientific Dionex Ultimate 3000 型高效液相色谱仪测定.

实验所用溶剂均采购于上海润捷化学试剂有限公 司，其中四氢呋喃使用前经无水处理.

\section{2 实验方法}

以紫杉醇(14a)、7,10-二甲氧基紫杉醇(15a)以及啞 唑烷型紫杉醇侧链(4S,5R)-3-苯甲酰基-2-(4-甲氧基苯 基)-4-苯基-5-噁唑啉羧酸 $(9 \mathbf{a})$ 及其前体 $(5 \mathbf{a}, 6 \mathbf{a}, 7 \mathbf{a}, 8 \mathbf{a})$ 为 例，其余衍生物的合成方法均与示例相同.

3.2.1 N-对甲氧基苯基- $(2 R, 3 S)$ - 苯基异丝氨酸乙酯 $(s y n-5 \mathbf{a})$ 的合成

将化合物 3 (50 mmol), $4 \mathbf{a}(52.5 \mathrm{mmol}), \mathrm{H}_{2} \mathrm{O}(2 \mathrm{~mL})$, $\mathrm{Cu}(\mathrm{OTf})_{2}(2.5 \mathrm{mmol})$ 加入 $500 \mathrm{~mL}$ 烧瓶中, 加入 $100 \mathrm{~mL}$ 
二氯甲烷 $(\mathrm{DCM})$ 溶解，搅拌 $30 \mathrm{~min}$. 之后将化合物 2 (75 $\mathrm{mmol}$ ) 溶于 $30 \mathrm{~mL} \mathrm{DCM}$ 后, 用恒压滴液漏斗缓慢滴入 $(30 \mathrm{~mL} / \mathrm{h})$. 继续常温摚拌 $0.5 \mathrm{~h}$ 后, 反应完毕, 加入 25 $\mathrm{mL}$ 水分液, 取 DCM 相, 干燥浓缩. 最后以 $V$ (石油醚) : $V($ 乙酸乙酯 $)=10: 1$ 为洗脱剂, 柱层析分离出 $4.11 \mathrm{~g}$ 白 色固体 syn-5a，总产率 $58 \%$, 顺式产物收率 $26 \%{ }^{\left[{ }^{[9]}\right.}$.

化合物 $s y n-\mathbf{5 b} \sim \mathbf{5 0}$ 的合成步骤与上述相同, 所得粗 产物经快速柱层析纯化后直接进入下一步合成.

\subsection{2 (2R,3S)-苯基异丝氨酸乙酯(6a)的合成}

将化合物 $s y n-\mathbf{5 a}(25 \mathrm{mmol})$ 加入 $500 \mathrm{~mL}$ 烧瓶中, 加 入 $60 \mathrm{~mL}$ 乙腈和 $45 \mathrm{~mL}$ 水将底物溶解. 再将 TCCA (13.75 mmol)加入烧瓶, 反应液变为紫黑色, 随后加入 1 $\mathrm{mol} / \mathrm{L} \mathrm{H}_{2} \mathrm{SO}_{4} 25 \mathrm{~mL}$, 室温下搅拌过夜. 反应液将慢慢地 由紫黑色浊液变为橙红色溶液, 最后在反应进行约 $12 \sim 16 \mathrm{~h}$ 时, 反应液变为黄色溶液. LC-MS 监测反应, 反应结束后加入 $\mathrm{DCM}(30 \mathrm{~mL} \times 3)$ 清洗反应液, 分液后 取水相. 向水相中加入 $2 \mathrm{~mol} / \mathrm{L} \mathrm{NaOH}$ 溶液调节 $\mathrm{pH}$ 值至 $9 \sim 10$, 随后加入乙酸乙酯萃 $(25 \mathrm{~mL} \times 2)$, 收集乙酸乙酯 相, 干燥浓缩后得到白色略带棕色固体. 将所得固体在 $V$ (石油醚) $: V($ 乙酸乙酯 $)=20: 1$ 条件下进行重结晶, 可以得到 $3.15 \mathrm{~g}$ 白色固体 $s y n-6 \mathrm{a}$, 收率 $60 \%{ }^{[9]}$.

化合物 $s y n-6 \mathbf{b} \sim 6 \mathbf{0}$ 的合成步骤与上述相同, 所得白 色略带棕色固体粗产物不经重结晶直接进入下一步合 成.

3.2.3 $\mathrm{N}$-苯甲酰基- $(2 R, 3 S)$ - 苯基异丝氨酸乙酯 $(7 \mathbf{a})$ 的 合成

将化合物 6a $(15 \mathrm{mmol})$ 加入 $250 \mathrm{~mL}$ 烧瓶中, 加入 $60 \mathrm{~mL}$ 乙酸乙酯和 $30 \mathrm{~mL}$ 饱和碳酸氢钠溶液溶解. 在冰 水浴下缓慢加入苯甲酰氯( $16.5 \mathrm{mmol}), 10 \mathrm{~min}$ 滴加完毕, 随后自然升温至室温, 继续反应 $2 \mathrm{~h}$. 薄层色谱(TLC)和 LC-MS 监测反应, 反应结束后分液, 取乙酸乙酯相, 干 燥浓缩, 得到白色固体. 将所得固体在 $V$ (石油醚)： $V($ 乙酸乙酯 $)=10: 1$ 条件下进行重结晶, 可以得到 3.85 $\mathrm{g}$ 白色固体 syn-7a, 收率 $82 \%{ }^{[13]}$. ${ }^{1} \mathrm{H}$ NMR $(400 \mathrm{MHz}$, $\left.\mathrm{CDCl}_{3}\right) \delta: 7.77(\mathrm{~d}, J=7.2 \mathrm{~Hz}, 2 \mathrm{H}), 7.51(\mathrm{t}, J=7.7 \mathrm{~Hz}, 1 \mathrm{H})$, $7.48 \sim 7.40(\mathrm{~m}, 4 \mathrm{H}), 7.37(\mathrm{t}, J=7.1 \mathrm{~Hz}, 2 \mathrm{H}), 7.31(\mathrm{~d}, J=$ $7.6 \mathrm{~Hz}, 1 \mathrm{H}), 7.00(\mathrm{~d}, J=9.0 \mathrm{~Hz}, 1 \mathrm{H}), 5.76$ (dd, $J=9.1,1.3$ $\mathrm{Hz}, 1 \mathrm{H}), 4.62(\mathrm{~s}, 1 \mathrm{H}), 4.36 \sim 4.23(\mathrm{~m}, 2 \mathrm{H}), 3.31(\mathrm{~s}, 1 \mathrm{H})$, $1.30(\mathrm{t}, J=7.1 \mathrm{~Hz}, 3 \mathrm{H}) ;{ }^{13} \mathrm{C}$ NMR $\left(101 \mathrm{MHz}, \mathrm{CDCl}_{3}\right) \delta$ : 173.0, 166.8, 138.7, 134.2, 131.8, 128.7, 128.7, 127.9, 127.1, 126.9, 73.3, 62.8, 54.7, 14.1. HRMS (ESI) calcd for $\mathrm{C}_{18} \mathrm{H}_{19} \mathrm{NNaO}_{4}[\mathrm{M}+\mathrm{Na}]^{+}:$336.1212, found 336.1225 .

化合物 7b $\sim 70$ 的合成步骤与 7a 相同.

$N$ - 苯甲酰基-(2R,3S)-3-(4-氯苯基)异丝氨酸乙酯 (7b): 白色固体 $3.97 \mathrm{~g}$, 收率 $80 \% .{ }^{1} \mathrm{H}$ NMR $(400 \mathrm{MHz}$,
$\left.\mathrm{CDCl}_{3}\right) \delta: 7.73(\mathrm{~d}, J=7.3 \mathrm{~Hz}, 2 \mathrm{H}), 7.48(\mathrm{t}, J=7.4 \mathrm{~Hz}, 1 \mathrm{H})$, $7.43 \sim 7.32(\mathrm{~m}, 4 \mathrm{H}), 7.28(\mathrm{~d}, J=8.5 \mathrm{~Hz}, 2 \mathrm{H}), 7.18(\mathrm{~d}, J=$ $9.0 \mathrm{~Hz}, 1 \mathrm{H}), 5.69$ (dd, $J=9.0,1.5 \mathrm{~Hz}, 1 \mathrm{H}), 4.54(\mathrm{dd}, J=$ $3.8,2.2 \mathrm{~Hz}, 1 \mathrm{H}), 4.31 \sim 4.18(\mathrm{~m}, 2 \mathrm{H}), 3.75(\mathrm{~d}, J=4.0 \mathrm{~Hz}$, $1 \mathrm{H}), 1.26$ (t, $J=7.1 \mathrm{~Hz}, 3 \mathrm{H}) ;{ }^{13} \mathrm{C}$ NMR $\left(101 \mathrm{MHz}, \mathrm{CDCl}_{3}\right)$ $\delta: 172.6,167.0,137.4,133.9,133.7,131.9,128.8,128.7$, 128.4, 127.1, 73.2, 62.7, 54.4, 14.1. HRMS (ESI) calcd for $\mathrm{C}_{18} \mathrm{H}_{19} \mathrm{NO}_{4} \mathrm{Cl}[\mathrm{M}+\mathrm{H}]^{+}: 348.1003$, found 348.1012 .

$N$-苯甲酰基-( $2 R, 3 S$ )-3-(4-甲基苯基)异丝氨酸乙酯 (7c): 白色固体 $4.10 \mathrm{~g}$, 收率 $83 \%$. ${ }^{1} \mathrm{H}$ NMR $(400 \mathrm{MHz}$, $\left.\mathrm{CDCl}_{3}\right) \delta: 7.76(\mathrm{~d}, J=7.7 \mathrm{~Hz}, 2 \mathrm{H}), 7.51(\mathrm{t}, J=7.0 \mathrm{~Hz}, 1 \mathrm{H})$, 7.43 (t, $J=7.5 \mathrm{~Hz}, 2 \mathrm{H}), 7.34$ (d, $J=7.6 \mathrm{~Hz}, 2 \mathrm{H}), 7.17$ (d, $J=7.7 \mathrm{~Hz}, 2 \mathrm{H}), 6.98(\mathrm{~d}, J=6.3 \mathrm{~Hz}, 1 \mathrm{H}), 5.72(\mathrm{~d}, J=9.0$ $\mathrm{Hz}, 1 \mathrm{H}), 4.60(\mathrm{~s}, 1 \mathrm{H}), 4.35 \sim 4.23(\mathrm{~m}, 2 \mathrm{H}), 3.34 \sim 3.30(\mathrm{~m}$, $1 \mathrm{H}), 2.33$ (s, 3H), 1.30 (t, $J=7.1 \mathrm{~Hz}, 3 \mathrm{H}) ;{ }^{13} \mathrm{C}$ NMR $(101$ $\left.\mathrm{MHz}, \mathrm{CDCl}_{3}\right) \delta: 173.0,166.8,137.6,135.7,134.2,131.7$, 129.4, 128.7, 127.1, 126.8, 73.4, 62.7, 54.6, 21.1, 14.1 . HRMS (ESI) calcd for $\mathrm{C}_{19} \mathrm{H}_{21} \mathrm{NO}_{4} \mathrm{Na}[\mathrm{M}+\mathrm{Na}]^{+}$: 350.1368 , found 350.1382 .

$N$-苯甲酰基-(2R,3S)-3-(4-甲氧基苯基)异丝氨酸乙 酯(7d): 白色固体 $4.18 \mathrm{~g}$, 收率 $83 \% .{ }^{1} \mathrm{H}$ NMR $(400 \mathrm{MHz}$, $\left.\mathrm{CDCl}_{3}\right) \delta: 7.75(\mathrm{~d}, J=7.2 \mathrm{~Hz}, 2 \mathrm{H}), 7.57 \sim 7.23(\mathrm{~m}, 5 \mathrm{H})$, $7.01(\mathrm{t}, J=9.3 \mathrm{~Hz}, 1 \mathrm{H}), 6.88(\mathrm{~d}, J=8.7 \mathrm{~Hz}, 2 \mathrm{H}), 5.69(\mathrm{t}$, $J=10.9 \mathrm{~Hz}, 1 \mathrm{H}), 4.58(\mathrm{~s}, 1 \mathrm{H}), 4.36 \sim 4.20(\mathrm{~m}, 2 \mathrm{H}), 3.78$ (s, 3H), $3.46(\mathrm{~s}, 1 \mathrm{H}), 1.29(\mathrm{t}, J=7.1 \mathrm{~Hz}, 3 \mathrm{H}) ;{ }^{13} \mathrm{C} \mathrm{NMR}$ $\left(101 \mathrm{MHz}, \mathrm{CDCl}_{3}\right) \delta: 173.0,172.7,166.8,159.2,154.6$, $134.2,134.0,132.1,131.8,131.7,130.9,128.9,128.7$, 128.6, 128.2, 127.1, 127.0, 126.6, 122.7, 73.4, 73.2, 62.8, $62.7,56.2,55.3,54.3,53.9,14.1$. HRMS (ESI) calcd for $\mathrm{C}_{19} \mathrm{H}_{21} \mathrm{NO}_{5} \mathrm{Na}[\mathrm{M}+\mathrm{Na}]^{+}: 366.1317$, found 366.1320 .

$N$-(4-甲氧基苯甲酰基)-( $2 R, 3 S$ )-3-苯基异丝氨酸乙 酯(7e): 白色固体 $4.05 \mathrm{~g}$, 收率 $80 \% .{ }^{1} \mathrm{H}$ NMR $(400 \mathrm{MHz}$, $\left.\mathrm{CDCl}_{3}\right) \delta: 7.74(\mathrm{~d}, J=8.7 \mathrm{~Hz}, 2 \mathrm{H}), 7.45(\mathrm{~d}, J=7.4 \mathrm{~Hz}$, 2H), 7.36 (t, $J=7.4 \mathrm{~Hz}, 2 \mathrm{H}), 7.29$ (t, $J=7.3 \mathrm{~Hz}, 1 \mathrm{H}), 6.92$ (d, $J=8.7 \mathrm{~Hz}, 2 \mathrm{H}), 6.91(\mathrm{~s}, 1 \mathrm{H}), 5.74(\mathrm{~d}, J=8.0 \mathrm{~Hz}, 1 \mathrm{H})$, $4.62(\mathrm{dd}, J=3.5,2.2 \mathrm{~Hz}, 1 \mathrm{H}), 4.34 \sim 4.22(\mathrm{~m}, 2 \mathrm{H}), 3.84$ (s, $3 \mathrm{H}), 3.34(\mathrm{~d}, J=3.8 \mathrm{~Hz}, 1 \mathrm{H}), 1.29(\mathrm{t}, J=7.1 \mathrm{~Hz}, 3 \mathrm{H}) ;{ }^{13} \mathrm{C}$ NMR (101 MHz, $\left.\mathrm{CDCl}_{3}\right) \delta: 173.0,166.3,162.4,138.9$, 128.9, 128.7, 127.9, 126.9, 126.4, 113.8, 73.4, 62.7, 55.5, 54.7, 14.1. HRMS (ESI) calcd for $\mathrm{C}_{19} \mathrm{H}_{21} \mathrm{NO}_{5} \mathrm{Na}[\mathrm{M}+$ $\mathrm{Na}]^{+}: 366.1317$, found 366.1321 .

$N$-(4-氯苯甲酰基)-(2R,3S)-3-苯基异丝氨酸乙酯(7f): 白色固体 $3.85 \mathrm{~g}$, 收率 79\%. ${ }^{1} \mathrm{H}$ NMR (400 $\left.\mathrm{MHz}, \mathrm{CDCl}_{3}\right)$ $\delta: 7.70(\mathrm{~d}, J=8.5 \mathrm{~Hz}, 2 \mathrm{H}), 7.51 \sim 7.33(\mathrm{~m}, 6 \mathrm{H}), 7.30(\mathrm{t}$, $J=7.2 \mathrm{~Hz}, 1 \mathrm{H}), 6.95(\mathrm{~d}, J=8.9 \mathrm{~Hz}, 1 \mathrm{H}), 5.73(\mathrm{~d}, J=8.2$ 
$\mathrm{Hz}, 1 \mathrm{H}), 4.61$ (s, 1H), $4.35 \sim 4.23(\mathrm{~m}, 2 \mathrm{H}), 3.27$ (d, $J=3.5$ $\mathrm{Hz}, 1 \mathrm{H}), 1.30$ (t, $J=7.1 \mathrm{~Hz}, 3 \mathrm{H}) ;{ }^{13} \mathrm{C}$ NMR $(101 \mathrm{MHz}$, $\left.\mathrm{CDCl}_{3}\right) \delta: 172.9,165.7,138.6,138.0,132.5,128.9,128.8$, $128.5,128.0,126.9,73.2,62.8,54.8,14.1$. HRMS (ESI) calcd for $\mathrm{C}_{18} \mathrm{H}_{19} \mathrm{NO}_{4} \mathrm{Cl}[\mathrm{M}+\mathrm{H}]^{+}:$348.1003, found 348.1012 .

$N$-(4-三氟甲基苯甲酰基)-( $2 R, 3 S$ )- 3 -苯基异丝氨酸 乙酯(7g): 白色固体 $4.21 \mathrm{~g}$, 收率 84\%. ${ }^{1} \mathrm{H}$ NMR (400 $\left.\mathrm{MHz}, \mathrm{CDCl}_{3}\right) \delta: 7.86(\mathrm{~d}, J=8.1 \mathrm{~Hz}, 2 \mathrm{H}), 7.68$ (d, $J=8.1$ $\mathrm{Hz}, 2 \mathrm{H}), 7.45$ (d, $J=7.4 \mathrm{~Hz}, 2 \mathrm{H}), 7.37$ (t, $J=7.3 \mathrm{~Hz}, 2 \mathrm{H})$, $7.31(\mathrm{t}, J=7.3 \mathrm{~Hz}, 1 \mathrm{H}), 7.10(\mathrm{~d}, J=9.0 \mathrm{~Hz}, 1 \mathrm{H}), 5.76(\mathrm{dd}$, $J=9.2,1.4 \mathrm{~Hz}, 1 \mathrm{H}), 4.62(\mathrm{~d}, J=1.9 \mathrm{~Hz}, 1 \mathrm{H}), 4.35 \sim 4.23$ (m, 2H), $3.40(\mathrm{~s}, 1 \mathrm{H}), 1.30(\mathrm{t}, J=7.1 \mathrm{~Hz}, 3 \mathrm{H}) ;{ }^{13} \mathrm{C} \mathrm{NMR}$ $\left(101 \mathrm{MHz}, \mathrm{CDCl}_{3}\right) \delta: 172.9,165.6,138.4,137.4,133.5$ (q, $J=33.4 \mathrm{~Hz}$ ), 128.8, 128.1, 127.6, 126.9, 125.7 (q, $J=3.6$ $\mathrm{Hz}), 123.6$ (q, $J=271.3 \mathrm{~Hz}$ ), 73.2, 62.8, 54.9, 14.1. HRMS (ESI) calcd for $\mathrm{C}_{19} \mathrm{H}_{18} \mathrm{~F}_{3} \mathrm{NO}_{4} \mathrm{Na}[\mathrm{M}+\mathrm{Na}]^{+}:$404.1124, found 404.1129 .

$N$-(4-二甲氨基苯甲酰基)-( $2 R, 3 S$ )-3-苯基异丝氨酸 乙酯(7h): 白色固体 $4.30 \mathrm{~g}$, 收率 $85 \%$. ${ }^{1} \mathrm{H}$ NMR (400 $\left.\mathrm{MHz}, \mathrm{CDCl}_{3}\right) \delta: 7.61(\mathrm{~d}, J=8.8 \mathrm{~Hz}, 2 \mathrm{H}), 7.37$ (d, $J=7.5$ $\mathrm{Hz}, 2 \mathrm{H}), 7.27$ (t, $J=7.4 \mathrm{~Hz}, 2 \mathrm{H}), 7.19$ (t, $J=7.3 \mathrm{~Hz}, 1 \mathrm{H})$, $6.78(\mathrm{~d}, J=9.0 \mathrm{~Hz}, 1 \mathrm{H}), 6.59(\mathrm{~d}, J=8.8 \mathrm{~Hz}, 2 \mathrm{H}), 5.67$ (d, $J=9.0 \mathrm{~Hz}, 1 \mathrm{H}), 4.54(\mathrm{~d}, J=2.1 \mathrm{~Hz}, 1 \mathrm{H}), 4.26 \sim 4.14(\mathrm{~m}$, 2H), $2.94(\mathrm{~s}, 6 \mathrm{H}), 1.21(\mathrm{t}, J=7.1 \mathrm{~Hz}, 3 \mathrm{H}) ;{ }^{13} \mathrm{C}$ NMR $(101$ $\left.\mathrm{MHz}, \mathrm{CDCl}_{3}\right) \delta: 172.0,165.7151 .67,138.2127 .6,127.6$ $126.7125 .9119 .7,110.1 \quad 72.5,61.6,53.7 \quad 39.1,13.1$. HRMS (ESI) calcd for $\mathrm{C}_{20} \mathrm{H}_{24} \mathrm{~N}_{2} \mathrm{O}_{4} \mathrm{Na}[\mathrm{M}+\mathrm{Na}]^{+}$: 379.1634, found 379.1640 .

$N$-[4-(3,5-二甲氧基苯甲酰基)]-(2R,3S)-3-苯基异丝 氨酸乙酯(7i): 白色固体 $3.98 \mathrm{~g}$, 收率 78\%. ${ }^{1} \mathrm{H}$ NMR (400 $\left.\mathrm{MHz}, \mathrm{CDCl}_{3}\right) \delta: 7.44(\mathrm{~d}, J=7.4 \mathrm{~Hz}, 2 \mathrm{H}), 7.36$ (t, $J=7.4$ $\mathrm{Hz}, 2 \mathrm{H}), 7.30$ (t, $J=7.2 \mathrm{~Hz}, 1 \mathrm{H}), 6.97(\mathrm{~d}, J=9.1 \mathrm{~Hz}, 1 \mathrm{H})$, $6.89(\mathrm{~d}, J=2.2 \mathrm{~Hz}, 2 \mathrm{H}), 6.58(\mathrm{t}, J=2.1 \mathrm{~Hz}, 1 \mathrm{H}), 5.72(\mathrm{dd}$, $J=9.1,1.4 \mathrm{~Hz}, 1 \mathrm{H}), 4.61(\mathrm{~d}, J=2.0 \mathrm{~Hz}, 1 \mathrm{H}), 4.36 \sim 4.23$ (m, 2H), $3.81(\mathrm{~s}, 6 \mathrm{H}), 1.31(\mathrm{t}, J=7.1 \mathrm{~Hz}, 3 \mathrm{H}) ;{ }^{13} \mathrm{C} \mathrm{NMR}$ $\left(101 \mathrm{MHz}, \mathrm{CDCl}_{3}\right) \delta: 172.9166 .7160 .97,138.7136 .4$ 128.7, 127.9, 126.9, 105.1 103.6, $73.362 .855 .654 .8,14.1$. HRMS (ESI) calcd for $\mathrm{C}_{20} \mathrm{H}_{23} \mathrm{NO}_{6} \mathrm{Na}[\mathrm{M}+\mathrm{Na}]^{+}$: 396.1424, found 396.1420 .

$N$-(2-㫙喃甲酰基)-( $2 R, 3 S)$-3-苯基异丝氨酸乙酯(7j): 白色固体 $4.00 \mathrm{~g}$, 收率 $83 \% .{ }^{1} \mathrm{H}$ NMR $\left(400 \mathrm{MHz}, \mathrm{CDCl}_{3}\right)$ $\delta: 7.49 \sim 7.42(\mathrm{~m}, 3 \mathrm{H}), 7.36(\mathrm{t}, J=7.4 \mathrm{~Hz}, 2 \mathrm{H}), 7.30(\mathrm{t}, J=$ $7.2 \mathrm{~Hz}, 1 \mathrm{H}), 7.20$ (d, $J=9.3 \mathrm{~Hz}, 1 \mathrm{H}), 7.10$ (d, $J=3.4 \mathrm{~Hz}$, $1 \mathrm{H}), 6.49(\mathrm{dd}, J=3.3,1.6 \mathrm{~Hz}, 1 \mathrm{H}), 5.71(\mathrm{~d}, J=9.4 \mathrm{~Hz}$,
1H), 4.59 (s, 1H), 4.29 (qd, $J=7.1,1.2 \mathrm{~Hz}, 2 \mathrm{H}), 3.35$ (s, $1 \mathrm{H}), 1.31(\mathrm{t}, J=7.1 \mathrm{~Hz}, 3 \mathrm{H}) ;{ }^{13} \mathrm{C}$ NMR $\left(101 \mathrm{MHz}, \mathrm{CDCl}_{3}\right)$ $\delta: 172.8157 .6147 .5144 .2138 .64,128.7,128.0,127.0$, $114.8,112.2,73.2,62.8,54.0,14.1$. HRMS (ESI) calcd for $\mathrm{C}_{16} \mathrm{H}_{18} \mathrm{NO}_{5}[\mathrm{M}+\mathrm{H}]^{+}:$304.1137, found 304.1134.

$N$-(2-噻吩甲酰基)-(2R,3S)-3-苯基异丝氨酸乙酯 (7k): 白色固体 $4.10 \mathrm{~g}$, 收率 83\%. ${ }^{1} \mathrm{H}$ NMR $(400 \mathrm{MHz}$, $\left.\mathrm{CDCl}_{3}\right) \delta: 7.52(\mathrm{~d}, J=3.4 \mathrm{~Hz}, 1 \mathrm{H}), 7.49 \sim 7.42(\mathrm{~m}, 3 \mathrm{H})$, $7.36(\mathrm{t}, J=7.4 \mathrm{~Hz}, 2 \mathrm{H}), 7.29(\mathrm{t}, J=7.2 \mathrm{~Hz}, 1 \mathrm{H}), 7.06(\mathrm{t}$, $J=4.9 \mathrm{~Hz}, 1 \mathrm{H}), 6.90(\mathrm{~d}, J=9.0 \mathrm{~Hz}, 1 \mathrm{H}), 5.71(\mathrm{~d}, J=9.1$ $\mathrm{Hz}, 1 \mathrm{H}), 4.60$ (s, 1H), 4.34 4.22 (m, 2H), 3.42 (d, $J=3.1$ $\mathrm{Hz}, 1 \mathrm{H}), 1.29$ (t, $J=7.1 \mathrm{~Hz}, 3 \mathrm{H}) ;{ }^{13} \mathrm{C}$ NMR $(101 \mathrm{MHz}$, $\left.\mathrm{CDCl}_{3}\right) \delta: 172.8,161.2,138.6,138.3,130.4,128.7,128.5$, 127.9, 127.7, 126.9, 73.2, 62.8, 54.7, 14.1. HRMS (ESI) calcd for $\mathrm{C}_{16} \mathrm{H}_{18} \mathrm{NO}_{4} \mathrm{~S}[\mathrm{M}+\mathrm{H}]^{+}$: 320.0982 , found 320.0988 .

$N$-环丙甲酰基-( $2 R, 3 S)$-3-苯基异丝氨酸乙酯(71): 白 色固体 $3.80 \mathrm{~g}$, 收率 78\%. ${ }^{1} \mathrm{H}$ NMR $\left(400 \mathrm{MHz}, \mathrm{CDCl}_{3}\right) \delta$ : 7.32 (d, $J=7.7 \mathrm{~Hz}, 2 \mathrm{H}), 7.27$ (t, $J=7.1 \mathrm{~Hz}, 2 \mathrm{H}), 7.21$ (t, $J=7.1 \mathrm{~Hz}, 1 \mathrm{H}), 6.47$ (d, $J=9.0 \mathrm{~Hz}, 1 \mathrm{H}), 5.49$ (d, $J=9.2$ $\mathrm{Hz}, 1 \mathrm{H}), 4.43$ (s, 1H), $4.25 \sim 4.13(\mathrm{~m}, 2 \mathrm{H}), 3.34$ (d, $J=3.5$ $\mathrm{Hz}, 1 \mathrm{H}), 1.34 \sim 1.28(\mathrm{~m}, 1 \mathrm{H}), 1.21(\mathrm{t}, J=7.1 \mathrm{~Hz}, 3 \mathrm{H})$, $0.88 \sim 0.82(\mathrm{~m}, 2 \mathrm{H}), 0.69 \sim 0.61(\mathrm{~m}, 2 \mathrm{H}) ;{ }^{13} \mathrm{C} \mathrm{NMR}(101$ $\left.\mathrm{MHz}, \mathrm{CDCl}_{3}\right) \delta: 172.0,171.9,138.0,127.6,126.7,125.9$, $72.4,61.5,53.5,13.6,13.1,6.4,6.4$. HRMS (ESI) calcd for $\mathrm{C}_{15} \mathrm{H}_{20} \mathrm{NO}_{4}[\mathrm{M}+\mathrm{H}]^{+}: 278.1392$, found 278.1364.

$N$-(4-三氟甲基苯磺酰基)-( $2 R, 3 S$ )-3-苯基异丝氨酸 乙酯(7m): 白色固体 $4.16 \mathrm{~g}$, 收率 $81 \% .{ }^{1} \mathrm{H}$ NMR (400 $\left.\mathrm{MHz}, \mathrm{CDCl}_{3}\right) \delta: 7.60$ (d, $\left.J=8.2 \mathrm{~Hz}, 2 \mathrm{H}\right), 7.39$ (d, $J=8.3$ $\mathrm{Hz}, 2 \mathrm{H}), 7.12 \sim 6.93(\mathrm{~m}, 5 \mathrm{H}), 5.88(\mathrm{~d}, J=9.8 \mathrm{~Hz}, 1 \mathrm{H})$, 4.84 (dd, $J=9.8,2.1 \mathrm{~Hz}, 1 \mathrm{H}), 4.27$ (s, 1H), 4.20 (q, $J=7.1$ $\mathrm{Hz}, 2 \mathrm{H}), 3.36$ (s, 1H), 1.26 (t, $J=7.1 \mathrm{~Hz}, 3 \mathrm{H}) ;{ }^{13} \mathrm{C}$ NMR $\left(101 \mathrm{MHz}, \mathrm{CDCl}_{3}\right) \delta: 171.0,142.9,135.4,132.9$ (q, $J=$ $33.5 \mathrm{~Hz}), 127.4,127.1,126.4,126.0,124.7$ (q, $J=3.6 \mathrm{~Hz}$ ), 122.1 (q, $J=273.0 \mathrm{~Hz}$ ), 73.6, 62.0, 58.5, 13.1. HRMS (ESI) calcd for $\mathrm{C}_{18} \mathrm{H}_{18} \mathrm{~F}_{3} \mathrm{NO}_{5} \mathrm{SNa}[\mathrm{M}+\mathrm{Na}]^{+}: 440.0755$, found 440.0793 .

$N$-(4-甲基苯磺酰基)-(2R,3S)-3-苯基异丝氨酸乙酯 (7n): 白色固体 $4.05 \mathrm{~g}$, 收率 $81 \% .{ }^{1} \mathrm{H}$ NMR $(400 \mathrm{MHz}$, $\left.\mathrm{CDCl}_{3}\right) \delta: 7.43(\mathrm{~d}, J=8.2 \mathrm{~Hz}, 2 \mathrm{H}), 7.13 \sim 7.02(\mathrm{~m}, 5 \mathrm{H})$, $6.99(\mathrm{~d}, J=8.0 \mathrm{~Hz}, 2 \mathrm{H}), 5.50(\mathrm{~d}, J=9.7 \mathrm{~Hz}, 1 \mathrm{H}), 4.79(\mathrm{dd}$, $J=9.7,2.2 \mathrm{~Hz}, 1 \mathrm{H}), 4.24(\mathrm{~s}, 1 \mathrm{H}), 4.19 \sim 4.08(\mathrm{~m}, 2 \mathrm{H})$, 3.19 (s, 1H), 2.25 (s, 3H), $1.23(\mathrm{t}, J=7.1 \mathrm{~Hz}, 3 \mathrm{H}) ;{ }^{13} \mathrm{C}$ NMR (101 MHz, $\left.\mathrm{CDCl}_{3}\right) \delta: 171.0,142.1,136.6,136.3$, $128.2,127.3,126.7,126.0,125.9,73.2,61.8,58.1,20.4$, 
13.0. HRMS (ESI) calcd for $\mathrm{C}_{18} \mathrm{H}_{22} \mathrm{NO}_{5} \mathrm{~S}[\mathrm{M}+\mathrm{H}]^{+}$: 364.1219 , found 364.1220 .

$N$-(4-乙酰胺基苯磺酰基)-(2R,3S)-3-苯基异丝氨酸 乙酯(7o): 白色固体 $4.25 \mathrm{~g}$, 收率 $82 \% .{ }^{1} \mathrm{H}$ NMR (400 MHz, DMSO- $\left.d_{6}\right) \delta: 10.12(\mathrm{~s}, 1 \mathrm{H}), 8.13(\mathrm{~d}, J=9.7 \mathrm{~Hz}, 1 \mathrm{H})$, $7.54 \sim 7.36(\mathrm{~m}, 4 \mathrm{H}), 7.21 \sim 7.03(\mathrm{~m}, 5 \mathrm{H}), 5.55(\mathrm{~d}, J=6.5$ $\mathrm{Hz}, 1 \mathrm{H}), 4.66(\mathrm{dd}, J=9.6,4.2 \mathrm{~Hz}, 1 \mathrm{H}), 4.16(\mathrm{t}, J=5.1$ $\mathrm{Hz}, 1 \mathrm{H}), 4.01 \sim 3.87$ (m, 2H), 2.04 (s, 3H), 1.08 (t, $J=7.1$ $\mathrm{Hz}, 3 \mathrm{H}) ;{ }^{13} \mathrm{C}$ NMR (101 MHz, DMSO- $\left.d_{6}\right) \delta: 171.3,168.7$, $142.1,138.2,135.3,127.5,127.4,127.3,126.9,117.9$, 74.4, 60.3, 60.2, 24.1, 13.9. HRMS (ESI) calcd for $\mathrm{C}_{19} \mathrm{H}_{22} \mathrm{~N}_{2} \mathrm{O}_{6} \mathrm{SNa}[\mathrm{M}+\mathrm{Na}]^{+}: 429.1096$, found 429.1084 .

3.2 .4 (4 $S, 5 R$ )-3-苯甲酰基-2-(4-甲氧基苯基)-4-苯基5 -缶唑啉羧酸乙酯 $(8 a)$ 的合成

将化合物 7a (12 mmol) 和对甲苯磺酸吡啶盐(PPTS, $1.2 \mathrm{mmol}$ )加入 $250 \mathrm{~mL}$ 烧瓶中, 加入 $100 \mathrm{~mL}$ 甲苯, 升温 至 $100{ }^{\circ} \mathrm{C}$, 摚拌 $10 \mathrm{~min}$ 至底物溶解. 之后向烧瓶中滴加 对甲氧基苯甲醛二甲缩酫 $\left(18 \mathrm{mmol}\right.$ ), 保持 $100{ }^{\circ} \mathrm{C}$ 反应 3 h. TLC 监测反应, 反应结束后降低至室温, 直接进行柱 层析. 以 $V$ (石油醚) $: V$ (乙酸乙酯 $)=8: 1$ 为洗脱剂, 可 以分离得到 $4.41 \mathrm{~g}$ 黄色油状液体 8a, 收率 85\%. ${ }^{1} \mathrm{H}$ NMR $\left(400 \mathrm{MHz}, \mathrm{CDCl}_{3}\right) \delta: 7.74 \sim 7.36(\mathrm{~m}, 2 \mathrm{H}), 7.36 \sim 7.28(\mathrm{~m}$, $4 \mathrm{H}), 7.35 \sim 7.12(\mathrm{~m}, 6 \mathrm{H}), 6.71 \sim 6.99(\mathrm{~m}, 3 \mathrm{H}), 5.35(\mathrm{~s}$, $1 \mathrm{H}), 4.85$ (s, 1H), 4.28 (q, $J=7.0 \mathrm{~Hz}, 2 \mathrm{H}), 3.81$ (s, 3H), $1.30(\mathrm{t}, J=7.0 \mathrm{~Hz}, 3 \mathrm{H}) ;{ }^{13} \mathrm{C}$ NMR $\left(101 \mathrm{MHz}, \mathrm{CDCl}_{3}\right) \delta$ : $170.8,169.9,160.0,139.0,136.8,134.0,129.7,128.8$, 128.6, 128.5, 128.2, 127.0, 113.6, 91.3, 82.4, 64.9, 62.0, 55.3, 14.2. HRMS (ESI) calcd for $\mathrm{C}_{26} \mathrm{H}_{26} \mathrm{NO}_{5}[\mathrm{M}+\mathrm{H}]^{+}$: 432.1844, found 432.1841 .

(4S,5R)-3-苯甲酰基-2-(4-甲氧基苯基)-4-(4-氯苯 基)-5-噁唑啉羧酸乙酯(8b): 黄色油状液体 $4.54 \mathrm{~g}$, 收率 $84 \% .{ }^{1} \mathrm{H}$ NMR (400 MHz, $\mathrm{CDCl}_{3}$ ) $\delta: 7.44 \sim 7.34(\mathrm{~m}, 2 \mathrm{H})$, $7.33 \sim 7.10(\mathrm{~m}, 9 \mathrm{H}), 6.94 \sim 6.76(\mathrm{~m}, 3 \mathrm{H}), 5.38(\mathrm{~s}, 1 \mathrm{H})$, 4.79 (s, 1H), 4.29 (q, $J=7.1 \mathrm{~Hz}, 2 \mathrm{H}), 3.81$ (s, 3H), 1.31 (t, $J=7.1 \mathrm{~Hz}, 3 \mathrm{H}) ;{ }^{13} \mathrm{C}$ NMR $\left(101 \mathrm{MHz}, \mathrm{CDCl}_{3}\right) \delta: 171.8$, $169.8,160.0,137.8,135.6,134.0,130.7,129.8,128.8$, 128.6, 128.3, 126.9, 113.6, 91.3, 82.0, 64.2, 62.1, 55.3, 14.2. HRMS (ESI) calcd for $\mathrm{C}_{26} \mathrm{H}_{25} \mathrm{NO}_{5} \mathrm{Cl}[\mathrm{M}+\mathrm{H}]^{+}$: 466.1362, found 466.1369 .

(4S,5R)-3-苯甲酰基-2-(4-甲氧基苯基)-4-(4-甲基苯 基)-5-腎唑啉羧酸乙酯(8c): 黄色油状液体 $4.25 \mathrm{~g}$, 收率 $82 \% .{ }^{1} \mathrm{H}$ NMR (400 MHz, $\left.\mathrm{CDCl}_{3}\right) \delta: 7.58 \sim 7.36(\mathrm{~m}, 2 \mathrm{H})$, $7.35(\mathrm{t}, J=7.5 \mathrm{~Hz}, 1 \mathrm{H}), 7.29$ (d, $J=7.5 \mathrm{~Hz}, 2 \mathrm{H}), 7.22$ (t, $J=7.1 \mathrm{~Hz}, 2 \mathrm{H}), 7.17 \sim 6.99(\mathrm{~m}, 4 \mathrm{H}), 6.96 \sim 6.70(\mathrm{~m}, 3 \mathrm{H})$, 5.34 (s, 1H), 4.82 (s, 1H), 4.28 (q, $J=7.0 \mathrm{~Hz}, 2 \mathrm{H}), 3.81$ (s,
3H), 2.35 (s, 3H), 1.30 (t, $J=7.1 \mathrm{~Hz}, 3 \mathrm{H}) ;{ }^{13} \mathrm{C}$ NMR (101 $\left.\mathrm{MHz}, \mathrm{CDCl}_{3}\right) \delta: 171.9,170.2,159.9,137.8,136.3,135.8$, 130.6, 130.1, 129.3, 128.8, 128.2, 127.0, 113.5, 91.2, 64.8, 61.9, 55.3, 21.1, 14.2. HRMS (ESI) calcd for $\mathrm{C}_{27} \mathrm{H}_{28} \mathrm{NO}_{5}$ $[\mathrm{M}+\mathrm{H}]^{+}:$446.1967, found 446.1980.

(4S,5R)-3-苯甲酰基-2-(4-甲氧基苯基)-4-(4-甲氧基 苯基)-5-噁唑啉羧酸乙酯(8d): 黄色油状液体 $4.46 \mathrm{~g}$, 收 率 84\%. ${ }^{1} \mathrm{H}$ NMR $\left(400 \mathrm{MHz}, \mathrm{CDCl}_{3}\right) \delta: 7.61 \sim 7.31(\mathrm{~m}$, $3 \mathrm{H}), 7.32 \sim 7.15(\mathrm{~m}, 6 \mathrm{H}), 7.14$ (br. s, $1 \mathrm{H}), 6.93 \sim 6.73(\mathrm{~m}$, 4H), $5.33(\mathrm{~s}, 1 \mathrm{H}), 4.79$ (d, $J=12.2 \mathrm{~Hz}, 1 \mathrm{H}), 4.29$ (qd, $J=$ $7.1,2.0 \mathrm{~Hz}, 2 \mathrm{H}), 3.99 \sim 3.74(\mathrm{~m}, 6 \mathrm{H}), 1.30(\mathrm{t}, J=6.9 \mathrm{~Hz}$, $3 \mathrm{H}) ;{ }^{13} \mathrm{C}$ NMR $\left(101 \mathrm{MHz}, \mathrm{CDCl}_{3}\right) \delta: 170.1,169.8,160.0$, $159.3,154.7,135.7,128.7,128.6,128.3,128.2,127.0$, 122.6, 113.9, 113.6, 111.9, 91.2, 82.0, 64.1, 62.0, 56.2, 55.3, 14.2. HRMS (ESI) calcd for $\mathrm{C}_{27} \mathrm{H}_{28} \mathrm{NO}_{6}[\mathrm{M}+\mathrm{H}]^{+}$: 462.1892, found 462.1885 .

(4S,5R)-3-(4-甲氧基苯甲酰基)-2-(4-甲氧基苯基)-4苯基-5-惡唑啉羧酸乙酯 $(8 \mathrm{e})$ : 黄色油状液体 $4.60 \mathrm{~g}$, 收 率 85\%. ${ }^{1} \mathrm{H}$ NMR $\left(400 \mathrm{MHz}, \mathrm{CDCl}_{3}\right) \delta: 7.47$ (d, $J=7.1$ $\mathrm{Hz}, 2 \mathrm{H}), 7.36 \sim 7.23(\mathrm{~m}, 7 \mathrm{H}), 6.96(\mathrm{~s}, 1 \mathrm{H}), 6.86(\mathrm{~d}, J=8.6$ $\mathrm{Hz}, 2 \mathrm{H}), 6.72(\mathrm{~d}, J=8.7 \mathrm{~Hz}, 2 \mathrm{H}), 5.45(\mathrm{~s}, 1 \mathrm{H}), 4.83(\mathrm{~d}, J=$ $1.4 \mathrm{~Hz}, 1 \mathrm{H}), 4.26$ (q, $J=7.1 \mathrm{~Hz}, 2 \mathrm{H}), 3.80$ (s, 3H), 3.74 (s, $3 \mathrm{H}), 1.29$ (t, $J=7.1 \mathrm{~Hz}, 3 \mathrm{H}) ;{ }^{13} \mathrm{C}$ NMR $\left(101 \mathrm{MHz}, \mathrm{CDCl}_{3}\right)$ $\delta: 171.8,170.1,161.6,159.9,139.6,130.3,129.3,128.7$, 128.7, 128.0, 127.8, 127.0, 113.5, 113.5, 91.2, 82.3, 65.3, 61.9, 55.3, 55.3, 14.2. HRMS (ESI) calcd for $\mathrm{C}_{27} \mathrm{H}_{28} \mathrm{NO}_{6}$ $[\mathrm{M}+\mathrm{H}]^{+}:$462.1892, found 462.1887 .

(4S,5R)-3-(4-氯苯甲酰基)-2-(4-甲氧基苯基)-4-苯 基-5-噁唑啉羧酸乙酯(8f): 黄色油状液体 $4.10 \mathrm{~g}$, 收率 $80 \%$. ${ }^{1} \mathrm{H}$ NMR $\left(400 \mathrm{MHz}, \mathrm{CDCl}_{3}\right) \delta: 7.43 \sim 7.34(\mathrm{~m}, 2 \mathrm{H})$, $7.34 \sim 7.09(\mathrm{~m}, 9 \mathrm{H}), 6.95 \sim 6.67(\mathrm{~m}, 3 \mathrm{H}), 5.38(\mathrm{~s}, 1 \mathrm{H})$, $4.79(\mathrm{~s}, 1 \mathrm{H}), 4.29$ (q, $J=7.1 \mathrm{~Hz}, 2 \mathrm{H}), 3.81(\mathrm{~s}, 3 \mathrm{H}), 1.31(\mathrm{t}$, $J=7.1 \mathrm{~Hz}, 3 \mathrm{H}) ;{ }^{13} \mathrm{C}$ NMR $\left(101 \mathrm{MHz}, \mathrm{CDCl}_{3}\right) \delta: 171.8$, $169.8,160.0,137.8,135.6,1334.0,130.7,129.8,128.8$, 128.6, 128.3, 126.9, 113.6, 91.3, 82.0, 64.2, 62.1, 55.29, $55.3,14.2$. HRMS (ESI) calcd for $\mathrm{C}_{26} \mathrm{H}_{25} \mathrm{NO}_{5} \mathrm{Cl}[\mathrm{M}+\mathrm{H}]^{+}$: 466.1362 , found 466.1375 .

(4S,5R)-3-(4-三氟甲基苯甲酰基)-2-(4-甲氧基苯 基)-4-苯基-5-慧唑啉羧酸乙酯 $(\mathbf{8 g})$ : 黄色油状液体 4.19 $\mathrm{g}$, 收率 80\%. ${ }^{1} \mathrm{H}$ NMR $\left(400 \mathrm{MHz}, \mathrm{CDCl}_{3}\right) \delta: 7.49(\mathrm{~d}, J=$ $8.1 \mathrm{~Hz}, 2 \mathrm{H}), 7.47 \sim 7.04$ (m, 9H), 6.84 (s, 3H), 5.30 (br. s, $1 \mathrm{H}), 4.88(\mathrm{~s}, 1 \mathrm{H}), 4.30$ (q, $J=7.0 \mathrm{~Hz}, 2 \mathrm{H}), 3.81(\mathrm{~s}, 3 \mathrm{H})$, $1.32(\mathrm{t}, J=7.1 \mathrm{~Hz}, 3 \mathrm{H}) ;{ }^{13} \mathrm{C} \mathrm{NMR}\left(101 \mathrm{MHz}, \mathrm{CDCl}_{3}\right) \delta$ : $169.9,160.1,139.1,138.8,132.2$ (q, $J=34.1 \mathrm{~Hz}), 129.4$, $128.8,128.3,127.3,127.1,126.0$ (q, $J=272.8 \mathrm{~Hz}), 125.3$ 
(q, $J=3.7 \mathrm{~Hz}), 113.6,91.3,81.9,65.0,62.1,55.3,14.2$. HRMS (ESI) calcd for $\mathrm{C}_{27} \mathrm{H}_{25} \mathrm{~F}_{3} \mathrm{NO}_{5}[\mathrm{M}+\mathrm{H}]^{+}:$500.1636, found 500.1647 .

(4S,5R)-3-(4-二甲氨基苯甲酰基)-2-(4-甲氧基苯 基)-4-苯基-5-噁唑啉羧酸乙酯 (8h): 无色油状液体 4.67 $\mathrm{g}$, 收率 85\%. ${ }^{1} \mathrm{H}$ NMR (400 MHz, $\left.\mathrm{CDCl}_{3}\right) \delta: 7.44(\mathrm{~d}, J=$ $8.5 \mathrm{~Hz}, 2 \mathrm{H}), 7.30 \sim 7.17(\mathrm{~m}, 7 \mathrm{H}), 6.96(\mathrm{~s}, 1 \mathrm{H}), 6.79(\mathrm{~d}, J=$ $8.7 \mathrm{~Hz}, 2 \mathrm{H}), 6.39$ (d, J=8.9 Hz, 2H), $5.46(\mathrm{~s}, 1 \mathrm{H}), 4.73(\mathrm{~d}$, $J=1.7 \mathrm{~Hz}, 1 \mathrm{H}), 4.24 \sim 4.12(\mathrm{~m}, 2 \mathrm{H}), 3.74(\mathrm{~s}, 3 \mathrm{H}), 2.86(\mathrm{~s}$, $6 \mathrm{H}), 1.21(\mathrm{t}, J=7.1 \mathrm{~Hz}, 3 \mathrm{H}) ;{ }^{13} \mathrm{C} \mathrm{NMR}\left(101 \mathrm{MHz}, \mathrm{CDCl}_{3}\right)$ $\delta: 171.4,169.2,158.7,151.1,139.0,129.7,128.5,127.7$, 127.6, 126.7, 126.0, 120.9, 112.4, 109.7, 90.2, 81.4, 64.5, 60.8, 54.3, 39.0, 13.1. HRMS (ESI) calcd for $\mathrm{C}_{28} \mathrm{H}_{31} \mathrm{~N}_{2} \mathrm{O}_{5}$ $[\mathrm{M}+\mathrm{H}]^{+}:$475.2256, found 475.2264 .

(4S,5R)-3-(3,5-二甲氧基苯甲酰基)-2-(4-甲氧基苯 基)-4-苯基-5-噁唑啉羧酸乙酯(8i): 无色油状液体 $4.24 \mathrm{~g}$, 收率 80\%. ${ }^{1} \mathrm{H}$ NMR $\left(400 \mathrm{MHz}, \mathrm{CDCl}_{3}\right) \delta: 7.69 \sim 7.39(\mathrm{~m}$, $2 \mathrm{H}), 7.37 \sim 7.16(\mathrm{~m}, 5 \mathrm{H}), 7.05 \sim 6.71(\mathrm{~m}, 3 \mathrm{H}), 6.43(\mathrm{~s}$, $3 \mathrm{H}), 5.35(\mathrm{~s}, 1 \mathrm{H}), 4.84(\mathrm{~s}, 1 \mathrm{H}), 4.29(\mathrm{q}, J=7.1 \mathrm{~Hz}, 2 \mathrm{H})$, $3.82(\mathrm{~s}, 3 \mathrm{H}), 3.44(\mathrm{~s}, 6 \mathrm{H}), 1.32(\mathrm{t}, J=7.1 \mathrm{~Hz}, 3 \mathrm{H}) ;{ }^{13} \mathrm{C}$ NMR $\left(101 \mathrm{MHz}, \mathrm{CDCl}_{3}\right) \delta: 171.8,170.0,160.4,159.9$, $137.3,130.1,128.8,128.7,128.0,127.0,113.5,104.6$, 104.1, 91.1, 62.0, 55.3, 55.1, 29.7, 14.2. HRMS (ESI) calcd for $\mathrm{C}_{28} \mathrm{H}_{30} \mathrm{NO}_{7}[\mathrm{M}+\mathrm{H}]^{+}:$: 492.2022, found 492.2040.

(4S,5R)-3-(2-呋喃甲酰基)-2-(4-甲氧基苯基)-4-苯 基-5-噁唑啉羧酸乙酯 $(\mathbf{8 j})$ : 无色油状液体 $4.28 \mathrm{~g}$, 收率 82\%. ${ }^{1} \mathrm{H}$ NMR (400 MHz, $\left.\mathrm{CDCl}_{3}\right) \delta: 7.48(\mathrm{~d}, J=8.4 \mathrm{~Hz}$, $2 \mathrm{H}), 7.38 \sim 7.27(\mathrm{~m}, 6 \mathrm{H}), 7.02(\mathrm{~s}, 1 \mathrm{H}), 6.93(\mathrm{~d}, J=3.2 \mathrm{~Hz}$, $1 \mathrm{H}), 6.86(\mathrm{~d}, J=8.8 \mathrm{~Hz}, 2 \mathrm{H}), 6.36(\mathrm{dd}, J=3.5,1.7 \mathrm{~Hz}$, $1 \mathrm{H}), 5.81(\mathrm{~d}, J=2.7 \mathrm{~Hz}, 1 \mathrm{H}), 4.91(\mathrm{~d}, J=2.3 \mathrm{~Hz}, 1 \mathrm{H}), 4.30$ (q, $J=7.1 \mathrm{~Hz}, 2 \mathrm{H}), 3.80(\mathrm{~s}, 3 \mathrm{H}), 1.31(\mathrm{t}, J=7.1 \mathrm{~Hz}, 3 \mathrm{H})$; ${ }^{13} \mathrm{C}$ NMR (101 MHz, $\left.\mathrm{CDCl}_{3}\right) \delta: 170.0,159.9,159.0,147.0$, $145.0,139.1,129.8,129.0,128.6,127.9,127.0,117.5$, $113.5,111.7,91.5,64.1,62.0,55.3,14.2$. HRMS (ESI) calcd for $\mathrm{C}_{24} \mathrm{H}_{24} \mathrm{NO}_{6}[\mathrm{M}+\mathrm{H}]^{+}:$: 22.1574 , found 422.1570 .

(4S,5R)-3-(2-噻吩甲酰基)-2-(4-甲氧基苯基)-4-苯 基-5-噁唑啉羧酸乙酯 $(8 \mathbf{k})$ : 无色油状液体 $4.33 \mathrm{~g}$, 收率 $83 \% .{ }^{1} \mathrm{H}$ NMR $\left(400 \mathrm{MHz}, \mathrm{CDCl}_{3}\right) \delta: 7.53(\mathrm{~d}, J=8.6 \mathrm{~Hz}$, 2H), $7.42(\mathrm{~d}, J=5.0 \mathrm{~Hz}, 1 \mathrm{H}), 7.38 \sim 7.30(\mathrm{~m}, 5 \mathrm{H}), 7.03(\mathrm{~d}$, $J=3.7 \mathrm{~Hz}, 1 \mathrm{H}), 6.99$ (s, 1H), $6.88(\mathrm{~d}, J=8.6 \mathrm{~Hz}, 2 \mathrm{H}), 6.84$ (t, $J=4.3 \mathrm{~Hz}, 1 \mathrm{H}), 5.73(\mathrm{~d}, J=2.1 \mathrm{~Hz}, 1 \mathrm{H}), 4.92(\mathrm{~d}, J=2.2$ $\mathrm{Hz}, 1 \mathrm{H}), 4.29$ (q, $J=7.5 \mathrm{~Hz}, 2 \mathrm{H}), 3.81$ (s, 3H), 1.29 (t, $J=$ $7.1 \mathrm{~Hz}, 3 \mathrm{H}) ;{ }^{13} \mathrm{C} \mathrm{NMR}\left(101 \mathrm{MHz}, \mathrm{CDCl}_{3}\right) \delta: 170.0,164.0$, $160.1,139.0,138.4,131.1,129.8,129.7,129.1,128.8$, $128.2,127.5,127.0,113.5,91.7,82.7,65.1,62.0,55.3$,
14.2. HRMS (ESI) calcd for $\mathrm{C}_{24} \mathrm{H}_{24} \mathrm{NO}_{5} \mathrm{~S}[\mathrm{M}+\mathrm{H}]^{+}$: 438.1365, found 438.1369 .

(4S,5R)-3-环丙甲酰基-2-(4-甲氧基苯基)-4-苯基-5噁唑啉羧酸乙酯 $(81)$ : 黄色油状液体 $3.89 \mathrm{~g}$, 收率 $75 \%$. ${ }^{1} \mathrm{H}$ NMR $\left(400 \mathrm{MHz}, \mathrm{CDCl}_{3}\right) \delta: 7.47 \sim 7.20(\mathrm{~m}, 7 \mathrm{H}), 6.81$ (d, $J=8.6 \mathrm{~Hz}, 2 \mathrm{H}), 6.65(\mathrm{~s}, 1 \mathrm{H}), 5.48(\mathrm{~s}, 1 \mathrm{H}), 4.78(\mathrm{~d}, J=$ $2.6 \mathrm{~Hz}, 1 \mathrm{H}), 4.33 \sim 4.14(\mathrm{~m}, 2 \mathrm{H}), 3.74(\mathrm{~s}, 3 \mathrm{H}), 1.26(\mathrm{t}, J=$ $6.9 \mathrm{~Hz}, 3 \mathrm{H}), 0.99 \sim 0.89(\mathrm{~m}, 1 \mathrm{H}), 0.89 \sim 0.75(\mathrm{~m}, 2 \mathrm{H})$, $0.69 \sim 0.42(\mathrm{~m}, 2 \mathrm{H}) ;{ }^{13} \mathrm{C}$ NMR $\left(101 \mathrm{MHz}, \mathrm{CDCl}_{3}\right) \delta$ : 171.9, 169.1, 138.3, 129.1, 127.8, 127.1, 112.8, 99.0, 90.3, 62.6, 61.1, 60.8, 54.3, 13.2, 12.4, 7.3. HRMS (ESI) calcd for $\mathrm{C}_{23} \mathrm{H}_{26} \mathrm{NO}_{5}[\mathrm{M}+\mathrm{H}]^{+}: 396.1747$, found 396.1743 .

(4S,5R)-3-(4-三氟甲基苯磺酰基)-2-(4-甲氧基苯 基)-4-苯基-5-惡唑啉羧酸乙酯(8m): 无色油状液体 4.08 $\mathrm{g}$, 收率 78\%. ${ }^{1} \mathrm{H}$ NMR $\left(400 \mathrm{MHz}, \mathrm{CDCl}_{3}\right) \delta:$ 7.55 7.43 (m, 4H), $7.33(\mathrm{~d}, J=8.6 \mathrm{~Hz}, 2 \mathrm{H}), 7.30 \sim 7.21(\mathrm{~m}, 5 \mathrm{H})$, $6.76(\mathrm{~d}, J=8.7 \mathrm{~Hz}, 2 \mathrm{H}), 6.37$ (s, 1H), 5.17 (d, $J=3.9 \mathrm{~Hz}$, $1 \mathrm{H}), 4.66(\mathrm{~d}, J=3.9 \mathrm{~Hz}, 1 \mathrm{H}), 4.06$ (q, $J=7.1 \mathrm{~Hz}, 2 \mathrm{H}), 3.73$ (s, 3H), 1.19 (t, $J=7.1 \mathrm{~Hz}, 3 \mathrm{H}) ;{ }^{13} \mathrm{C}$ NMR $(101 \mathrm{MHz}$, $\left.\mathrm{CDCl}_{3}\right) \delta: 167.9,159.6,140.8,137.5,133.5$ (q, $J=33.4$ $\mathrm{Hz}), 128.2,127.8,127.5,127.2,127.0,126.2,124.8$ (q, $J=$ $3.9 \mathrm{~Hz}), 119.4$ (q, $J=273.1 \mathrm{~Hz}), 112.8,92.1,81.3,64.2$, 61.0, 54.3, 13.0. HRMS (ESI) calcd for $\mathrm{C}_{26} \mathrm{H}_{25} \mathrm{~F}_{3} \mathrm{NO}_{6} \mathrm{~S}$ $[\mathrm{M}+\mathrm{H}]^{+}: 536.1366$, found 536.1377 .

(4S,5R)-3-(4-甲基苯磺酰基)-2-(4-甲氧基苯基)-4-苯 基-5-噁唑啉羧酸乙酯 $(\mathbf{8 n})$ : 无色油状液体 $4.27 \mathrm{~g}$, 收率 83\%. ${ }^{1} \mathrm{H}$ NMR (400 MHz, $\left.\mathrm{CDCl}_{3}\right) \delta: 7.53(\mathrm{~d}, J=8.2 \mathrm{~Hz}$, $2 \mathrm{H}), 7.41(\mathrm{~d}, J=8.6 \mathrm{~Hz}, 2 \mathrm{H}), 7.30 \sim 7.13(\mathrm{~m}, 7 \mathrm{H}), 6.81(\mathrm{~d}$, $J=8.7 \mathrm{~Hz}, 2 \mathrm{H}), 6.36(\mathrm{~s}, 1 \mathrm{H}), 5.04(\mathrm{~d}, J=4.5 \mathrm{~Hz}, 1 \mathrm{H}), 4.57$ (d, $J=4.5 \mathrm{~Hz}, 1 \mathrm{H}), 4.03 \sim 3.92(\mathrm{~m}, 2 \mathrm{H}), 3.74$ (s, 3H), 2.34 (s, 3H), $1.14(\mathrm{t}, J=7.1 \mathrm{~Hz}, 3 \mathrm{H}) ;{ }^{13} \mathrm{C}$ NMR $(101 \mathrm{MHz}$, $\left.\mathrm{CDCl}_{3}\right) \delta: 167.9,159.3,143.1,137.8,133.5,128.6,128.3$, 127.8, 127.6, 127.1, 126.1, 112.7, 92.1, 81.3, 64.5, 60.8, 54.3, 20.5, 13.0. HRMS (ESI) calcd for $\mathrm{C}_{26} \mathrm{H}_{28} \mathrm{NO}_{6} \mathrm{~S}$ $[\mathrm{M}+\mathrm{H}]^{+}:$482.1638, found 482.1645 .

(4S,5R)-3-(4-乙酰胺基苯磺酰基)-2-(4-甲氧基苯 基)-4-苯基-5-噁唑啉羧酸乙酯(80): 无色油状液体 3.82 $\mathrm{g}$, 收率 73\%. ${ }^{1} \mathrm{H}$ NMR $\left(400 \mathrm{MHz}, \mathrm{CDCl}_{3}\right) \delta: 7.83(\mathrm{~s}, 1 \mathrm{H})$, $7.67 \sim 7.55(\mathrm{~m}, 4 \mathrm{H}), 7.47(\mathrm{~d}, J=8.6 \mathrm{~Hz}, 2 \mathrm{H}), 7.39 \sim 7.26$ (m, 5H), 6.87 (d, $J=8.6 \mathrm{~Hz}, 2 \mathrm{H}), 6.40(\mathrm{~s}, 1 \mathrm{H}), 5.11(\mathrm{~d}, J=$ $4.4 \mathrm{~Hz}, 1 \mathrm{H}), 4.66(\mathrm{~d}, J=4.4 \mathrm{~Hz}, 1 \mathrm{H}), 4.13 \sim 4.01(\mathrm{~m}, 2 \mathrm{H})$, 3.80 (s, 3H), $2.12(\mathrm{~s}, 3 \mathrm{H}), 1.21(\mathrm{t}, J=7.1 \mathrm{~Hz}, 3 \mathrm{H}) ;{ }^{13} \mathrm{C}$ NMR $\left(101 \mathrm{MHz}, \mathrm{CDCl}_{3}\right) \delta: 168.9,168.9,160.4,142.8$, $138.7,131.6,129.2,129.1,128.9,128.7,128.3,127.1$, $119.1,113.8,93.2,82.3,65.6,62.0,55.3,24.6,14.0$. 
HRMS (ESI) calcd for $\mathrm{C}_{27} \mathrm{H}_{29} \mathrm{~N}_{2} \mathrm{O}_{7} \mathrm{~S}[\mathrm{M}+\mathrm{H}]^{+}$: 525.1695, found 525.1680 .

3.2.5 (4 $S, 5 R$ )-3-苯甲酰基-2-(4-甲氧基苯基)-4-苯基5 -嗅唑啉羧酸 $(9 a)$ 的合成

向化合物 8a (11 mmol)加入 $40 \mathrm{~mL}$ 甲醇和 $20 \mathrm{~mL}$ 水, 再加入 $\mathrm{NaOH}(33 \mathrm{mmol})$, 室温摚拌 $2 \mathrm{~h}$. TLC 监测反应, 反应结束后真空旋干溶剂, 再向得到的残余物中加入 $25 \mathrm{~mL}$ 水溶解. 随后向溶液中加入 $1 \mathrm{~mol} / \mathrm{L} \mathrm{HCl}$ 调节 $\mathrm{pH}$ 值至 3 4, 有大量白色固体析出, 过滤、干燥得到 $4.21 \mathrm{~g}$ 黄色固体 9a, 收率 95\% ${ }^{[13]}$ 。 ${ }^{1} \mathrm{H}$ NMR $(400 \mathrm{MHz}$, DMSO- $\left.d_{6}\right) \delta: 13.64(\mathrm{~s}, 1 \mathrm{H}), 7.55 \sim 7.35(\mathrm{~m}, 2 \mathrm{H}), 7.35 \sim$ $7.12(\mathrm{~m}, 10 \mathrm{H}), 6.90(\mathrm{~d}, J=7.9 \mathrm{~Hz}, 2 \mathrm{H}), 6.63(\mathrm{~s}, 1 \mathrm{H}), 5.30$ (s, 1H), $4.90(\mathrm{~s}, 1 \mathrm{H}), 3.76(\mathrm{~s}, 3 \mathrm{H}) .{ }^{13} \mathrm{C}$ NMR $(101 \mathrm{MHz}$, DMSO- $\left.d_{6}\right) \delta: 170.9,159.4,138.4,135.7,132.4,130.5$, 129.6, 128.7, 128.4, 128.4, 126.3, 113.5, 90.0, 55.1. HRMS (ESI) calcd for $\mathrm{C}_{24} \mathrm{H}_{21} \mathrm{NO}_{5} \mathrm{Na}[\mathrm{M}+\mathrm{Na}]^{+}: 426.1317$, found 426.1310 .

\subsection{6 紫杉醇 $(\mathbf{1 4 a})$ 的合成}

将化合物 $10(0.1 \mathrm{mmol}) 、$ 化合物 $9 \mathbf{a}(0.23 \mathrm{mmol})$ 、 二甲氨基吡啶(DMAP, $0.23 \mathrm{mmol}$ )和 $2.5 \mathrm{~mL}$ 无水甲苯加 入 $50 \mathrm{~mL}$ 烧瓶中, 加热至 $70{ }^{\circ} \mathrm{C}$ 使反应液溶清, 随后加 入 DCC ( $0.33 \mathrm{mmol})$, 搅拌 $1 \mathrm{~h}$. TCL 和 LC-MS 监测反应, 反应结束后过滤, 收集滤液. 最后以 $V$ (石油醚) $: V($ 乙 酸乙酯 $)=2: 1$ 为洗脱剂柱层析分离得到白色固体 12a, 收率为 $95 \%$. 向所得化合物 12a $(0.1 \mathrm{mmol})$ 中加入 $3 \mathrm{~mL}$ 无水乙醇, 呈白色浊液, 再向反应液中加入 $20 \% \sim 30 \%$ 的 $\mathrm{HCl} / \mathrm{EtOH}$ 溶液 $0.5 \mathrm{~mL}$, 室温下搅拌 $24 \mathrm{~h}$, 反应液澄 清. LC-MS 监测反应, 反应结束后加入 $1.5 \mathrm{~mL}$ 饱和 $\mathrm{NaHCO}_{3}$ 溶液、 $0.5 \mathrm{~mL}$ 水和 $10 \mathrm{~mL}$ 乙酸乙酯萃取分液, 水相再用 $10 \mathrm{~mL}$ 乙酸乙酯反萃, 合并乙酸乙酯相, 干燥 浓缩. 最后以 $V$ (二氯甲烷) $: V$ (甲醇 $)=20: 1$ 为洗脱剂 柱层析分离得到 $46 \mathrm{mg}$ 白色固体 14a, 收率为 $57 \%$.

3'-(4-氯苯基)紫杉醇(14b): 白色固体 $26 \mathrm{mg}$, 产率 48\%. m.p. $159 \sim 160{ }^{\circ} \mathrm{C} ;{ }^{1} \mathrm{H}$ NMR (400 MHz, $\left.\mathrm{CDCl}_{3}\right) \delta$ : 7.98 (d, $J=7.5 \mathrm{~Hz}, 2 \mathrm{H}), 7.66$ (d, $J=7.4 \mathrm{~Hz}, 2 \mathrm{H}), 7.54$ (t, $J=7.4 \mathrm{~Hz}, 1 \mathrm{H}), 7.49 \sim 7.29(\mathrm{~m}, 9 \mathrm{H}), 6.97(\mathrm{~d}, J=8.8 \mathrm{~Hz}$, $1 \mathrm{H}), 6.25$ (s, 1H), 6.13 (t, $J=8.6 \mathrm{~Hz}, 1 \mathrm{H}), 5.67$ (d, $J=8.8$ $\mathrm{Hz}, 1 \mathrm{H}), 5.59(\mathrm{~d}, J=7.0 \mathrm{~Hz}, 1 \mathrm{H}), 4.90(\mathrm{~d}, J=8.7 \mathrm{~Hz}, 1 \mathrm{H})$, 4.56 (s, 1H), 4.39 (dd, $J=10.3,8.52 \mathrm{~Hz}, 1 \mathrm{H}), 4.22$ (d, $J=$ $8.4 \mathrm{~Hz}, 1 \mathrm{H}), 4.09$ (d, $J=8.6 \mathrm{~Hz}, 1 \mathrm{H}), 3.79(\mathrm{~d}, J=7.0 \mathrm{~Hz}$, $1 \mathrm{H}), 3.75 \sim 3.59(\mathrm{~m}, 1 \mathrm{H}), 2.60 \sim 2.44(\mathrm{~m}, 2 \mathrm{H}), 2.31(\mathrm{dd}$, $J=15.1,9.2 \mathrm{~Hz}, 1 \mathrm{H}), 2.13$ (s, 3H), 2.14 (s, 3H), 2.11 $2.08(\mathrm{~m}, 1 \mathrm{H}), 1.97(\mathrm{~s}, 3 \mathrm{H}), 1.86 \sim 1.80(\mathrm{~m}, 1 \mathrm{H}), 1.61(\mathrm{~s}$, $3 \mathrm{H}), 1.14(\mathrm{~s}, 3 \mathrm{H}), 1.06(\mathrm{~s}, 3 \mathrm{H}) ;{ }^{13} \mathrm{C}$ NMR (101 MHz, $\left.\mathrm{CDCl}_{3}\right) \delta: 202.6,171.4,170.1,168.5,166.0,141.0,136.4$,
$133.3,132.8,132.6,132.0,131.1,129.0,128.2,128.1$, $127.8,127.7,127.7,126.1,83.4,80.2,78.2,76.2,75.4$, 74.5, 73.8, 72.2, 71.6, 71.2, 57.6, 53.6, 44.8, 42.1, 35.0, 34.6, 28.7, 25.74, 21.6, 20.5, 19.8, 14.3, 8.5. HRMS (ESI) calcd for $\mathrm{C}_{47} \mathrm{H}_{51} \mathrm{ClNO}_{14}[\mathrm{M}+\mathrm{H}]^{+}$: 888.2998, found 888.2982.

3'-( $N$-对甲氧基苯甲酰基)紫杉醇(14e): 白色固体 27 $\mathrm{mg}$, 产率 51\%. m.p. $158 \sim 159{ }^{\circ} \mathrm{C} ;{ }^{1} \mathrm{H}$ NMR $(400 \mathrm{MHz}$, $\left.\mathrm{CDCl}_{3}\right) \delta: 8.05(\mathrm{~d}, J=7.6 \mathrm{~Hz}, 2 \mathrm{H}), 7.63(\mathrm{~d}, J=8.6 \mathrm{~Hz}$, 2H), $7.54(\mathrm{t}, J=7.3 \mathrm{~Hz}, 1 \mathrm{H}), 7.46 \sim 7.36(\mathrm{~m}, 4 \mathrm{H}), 7.33(\mathrm{t}$, $J=7.4 \mathrm{~Hz}, 2 \mathrm{H}), 7.26(\mathrm{t}, J=7.1 \mathrm{~Hz}, 1 \mathrm{H}), 6.90$ (d, $J=8.7$ $\mathrm{Hz}, 1 \mathrm{H}), 6.79$ (d, $J=8.6 \mathrm{~Hz}, 2 \mathrm{H}), 6.19$ (s, 1H), 6.13 (t, $J=$ $8.7 \mathrm{~Hz}, 1 \mathrm{H}), 5.68(\mathrm{~d}, J=8.5 \mathrm{~Hz}, 1 \mathrm{H}), 5.59(\mathrm{~d}, J=7.0 \mathrm{~Hz}$, $1 \mathrm{H}), 4.86$ (d, $J=9.2 \mathrm{~Hz}, 1 \mathrm{H}), 4.69$ (s, 1H), 4.31 (t, $J=7.5$ $\mathrm{Hz}, 1 \mathrm{H}), 4.21(\mathrm{~d}, J=8.4 \mathrm{~Hz}, 1 \mathrm{H}), 4.11(\mathrm{~d}, J=8.4 \mathrm{~Hz}, 1 \mathrm{H})$, $3.74(\mathrm{~s}, 3 \mathrm{H}), 3.73 \sim 3.68(\mathrm{~m}, 1 \mathrm{H}), 2.51 \sim 2.39(\mathrm{~m}, 2 \mathrm{H})$, $2.30(\mathrm{~s}, 3 \mathrm{H}), 2.27 \sim 2.17(\mathrm{~m}, 2 \mathrm{H}), 2.15(\mathrm{~s}, 3 \mathrm{H}), 1.84 \sim 1.79$ (m, 1H), $1.71(\mathrm{~s}, 3 \mathrm{H}), 1.60(\mathrm{~s}, 3 \mathrm{H}), 1.15(\mathrm{~s}, 3 \mathrm{H}), 1.06(\mathrm{~s}$, $3 \mathrm{H}) ;{ }^{13} \mathrm{C}$ NMR (101 MHz, $\mathrm{CDCl}_{3}$ ) $\delta: 202.6,171.7,170.2$, $169.4,166.0,165.7,161.5,141.0,137.1,132.7,132.1$, $129.2,128.2,128.0,127.9,127.7,127.3,126.0,124.8$, $112.8,83.4,80.1,78.0,76.2,75.5,74.6,73.9,72.4,71.2$, 71.2, 57.6, 54.4, 54.1, 44.6, 42.1, 34.7, 34.6, 25.8, 21.6, $20.8,19.8,13.8,8.5$. HRMS (ESI) calcd for $\mathrm{C}_{48} \mathrm{H}_{54} \mathrm{NO}_{15}$ $[\mathrm{M}+\mathrm{H}]^{+}:$: 884.3493, found 884.3540 ..

$3^{\prime}-(\mathrm{N}$-对三氟甲基苯甲酰基)紫杉醇(14g): 白色固体 $19 \mathrm{mg}$, 产率 50\%. m.p. $163 \sim 165{ }^{\circ} \mathrm{C} ;{ }^{1} \mathrm{H}$ NMR (400 $\left.\mathrm{MHz}, \mathrm{CDCl}_{3}\right) \delta: 8.07$ (d, $\left.J=7.4 \mathrm{~Hz}, 2 \mathrm{H}\right), 7.78$ (d, $J=8.1$ $\mathrm{Hz}, 2 \mathrm{H}), 7.59$ (d, $J=8.1 \mathrm{~Hz}, 2 \mathrm{H}), 7.54(\mathrm{t}, J=7.5 \mathrm{~Hz}, 1 \mathrm{H})$, $7.47 \sim 7.29(\mathrm{~m}, 7 \mathrm{H}), 7.00(\mathrm{~d}, J=8.8 \mathrm{~Hz}, 1 \mathrm{H}), 6.20(\mathrm{~s}, 1 \mathrm{H})$, $6.16(\mathrm{~d}, J=8.4 \mathrm{~Hz}, 1 \mathrm{H}), 5.72(\mathrm{~d}, J=8.9 \mathrm{~Hz}, 1 \mathrm{H}), 5.60(\mathrm{~d}$, $J=7.0 \mathrm{~Hz}, 1 \mathrm{H}), 4.87(\mathrm{~d}, J=8.4 \mathrm{~Hz}, 1 \mathrm{H}), 4.73(\mathrm{~s}, 1 \mathrm{H})$, $4.37 \sim 4.28(\mathrm{~m}, 1 \mathrm{H}), 4.24(\mathrm{~d}, J=8.4 \mathrm{~Hz}, 1 \mathrm{H}), 4.12(\mathrm{~d}, J=$ $8.4 \mathrm{~Hz}, 1 \mathrm{H}), 3.73$ (d, $J=6.9 \mathrm{~Hz}, 1 \mathrm{H}), 3.43(\mathrm{~s}, 1 \mathrm{H}), 2.53 \sim$ $2.42(\mathrm{~m}, 1 \mathrm{H}), 2.40(\mathrm{~d}, J=3.7 \mathrm{~Hz}, 1 \mathrm{H}), 2.31(\mathrm{~s}, 3 \mathrm{H}), 2.28 \sim$ $2.19(\mathrm{~m}, 2 \mathrm{H}), 2.17(\mathrm{~s}, 3 \mathrm{H}), 1.85 \sim 1.77(\mathrm{~m}, 1 \mathrm{H}), 1.71(\mathrm{~s}$, $3 \mathrm{H}), 1.62(\mathrm{~s}, 3 \mathrm{H}), 1.18(\mathrm{~s}, 3 \mathrm{H}), 1.08(\mathrm{~s}, 3 \mathrm{H}) ;{ }^{13} \mathrm{C} \mathrm{NMR}$ $\left(101 \mathrm{MHz}, \mathrm{CDCl}_{3}\right) \delta: 202.5,171.6,170.3,169.3,166.0$, $164.7,140.8,136.6,135.9,135.3,132.8,132.3,129.2$, 128.1, 127.7, 127.5, 126.5, 126.0, 124.7 (q, $J=3.9 \mathrm{~Hz}$ ), $83.4,80.2,78.1,76.2,74.5,71.9,71.5,71.2,57.6,54.0$, 44.6, 42.2, 34.6, 28.7, 25.9, 21.6, 20.8, 19.8, 13.8, 8.5. HRMS (ESI) calcd for $\mathrm{C}_{48} \mathrm{H}_{51} \mathrm{~F}_{3} \mathrm{NO}_{14}[\mathrm{M}+\mathrm{H}]^{+}$: 922.3262 , found 922.3292 .

3'-( $N$-对二甲氨基苯甲酰基)紫杉醇(14h): 白色固体 
$27 \mathrm{mg}$, 产率 60\%. m.p. $166 \sim 169{ }^{\circ} \mathrm{C} ;{ }^{1} \mathrm{H}$ NMR (400 $\left.\mathrm{MHz}, \mathrm{CDCl}_{3}\right) \delta: 8.06(\mathrm{~d}, J=7.7 \mathrm{~Hz}, 2 \mathrm{H}), 7.58(\mathrm{~d}, J=8.7$ $\mathrm{Hz}, 2 \mathrm{H}), 7.54$ (t, $J=7.3 \mathrm{~Hz}, 1 \mathrm{H}), 7.44(\mathrm{t}, J=8.0 \mathrm{~Hz}, 2 \mathrm{H})$, 7.39 (d, $J=7.8 \mathrm{~Hz}, 2 \mathrm{H}), 7.33(\mathrm{t}, J=7.5 \mathrm{~Hz}, 2 \mathrm{H}), 7.26$ (t, $J=7.1 \mathrm{~Hz}, 1 \mathrm{H}), 6.74(\mathrm{~d}, J=8.6 \mathrm{~Hz}, 1 \mathrm{H}), 6.54(\mathrm{~d}, J=8.7$ $\mathrm{Hz}, 2 \mathrm{H}), 6.20(\mathrm{~s}, 1 \mathrm{H}), 6.13(\mathrm{t}, J=8.8 \mathrm{~Hz}, 1 \mathrm{H}), 5.68(\mathrm{~d}, J=$ $8.6 \mathrm{~Hz}, 1 \mathrm{H}), 5.60(\mathrm{~d}, J=6.9 \mathrm{~Hz}, 1 \mathrm{H}), 4.88(\mathrm{~d}, J=9.2 \mathrm{~Hz}$, $1 \mathrm{H}), 4.71(\mathrm{~d}, J=2.5 \mathrm{~Hz}, 1 \mathrm{H}), 4.33(\mathrm{dd}, J=8.9,8.4 \mathrm{~Hz}$, $1 \mathrm{H}), 4.22(\mathrm{~d}, J=8.4 \mathrm{~Hz}, 1 \mathrm{H}), 4.13(\mathrm{~d}, J=8.3 \mathrm{~Hz}, 1 \mathrm{H}), 3.72$ (d, $J=6.9 \mathrm{~Hz}, 1 \mathrm{H}), 2.93$ (s, 6H), $2.54 \sim 2.35(\mathrm{~m}, 2 \mathrm{H}), 2.32$ (s, 3H), $2.29 \sim 2.17(\mathrm{~m}, 2 \mathrm{H}), 2.16(\mathrm{~s}, 3 \mathrm{H}), 1.86 \sim 1.79(\mathrm{~m}$, 1H), 1.73 (s, 3H), 1.61 (s, 3H), $1.16(\mathrm{~s}, 3 \mathrm{H}), 1.07$ (s, 3H). ${ }^{13} \mathrm{C}$ NMR (101 MHz, $\left.\mathrm{CDCl}_{3}\right) \delta: 202.7,171.8,170.3,169.4$, $166.2,165.9,151.8,141.2,137.4,132.7,132.0,129.2$, $128.2,127.9,127.7,127.6,127.2,126.0,119.0,110.0$, 83.4, 80.1, 78.0, 74.6, 73.9, 72.7, 71.2, 57.6, 54.3, 44.6, 42.2, 39.1, 34.7, 34.6, 25.8, 21.6, 20.9, 19.9, 13.9, 8.5. HRMS (ESI) calcd for $\mathrm{C}_{49} \mathrm{H}_{57} \mathrm{~N}_{2} \mathrm{O}_{14}[\mathrm{M}+\mathrm{H}]^{+}$: 897.3810, found 897.3781 .

$3^{\prime}$-[ $N$-(3,5-二甲氧基苯甲酰基)]紫杉醇(14i): 白色固 体 $17 \mathrm{mg}$, 产率 46\%. m.p. 148 149 ${ }^{\circ} \mathrm{C} ;{ }^{1} \mathrm{H}$ NMR (400 $\left.\mathrm{MHz}, \mathrm{CDCl}_{3}\right) \delta: 8.10(\mathrm{~d}, J=7.6 \mathrm{~Hz}, 2 \mathrm{H}), 7.54$ (t, $J=7.4$ $\mathrm{Hz}, 1 \mathrm{H}), 7.44$ (t, $J=7.4 \mathrm{~Hz}, 2 \mathrm{H}), 7.42 \sim 7.32(\mathrm{~m}, 4 \mathrm{H}), 7.28$ (t, $J=6.9 \mathrm{~Hz}, 1 \mathrm{H}), 6.87(\mathrm{~d}, J=8.9 \mathrm{~Hz}, 1 \mathrm{H}), 6.76(\mathrm{~s}, 2 \mathrm{H})$, $6.48(\mathrm{~s}, 1 \mathrm{H}), 6.20(\mathrm{~s}, 1 \mathrm{H}), 6.18(\mathrm{t}, J=9.2 \mathrm{~Hz}, 1 \mathrm{H}), 5.73(\mathrm{~d}$, $J=8.7 \mathrm{~Hz}, 1 \mathrm{H}), 5.60(\mathrm{~d}, J=7.1 \mathrm{~Hz}, 1 \mathrm{H}), 4.87(\mathrm{~d}, J=9.2$ Hz, 1H), 4.72 (br.s, 1H), 4.38 4.29 (m, 1H), 4.23 (d, $J=$ $8.3 \mathrm{~Hz}, 1 \mathrm{H}), 4.12(\mathrm{~d}, J=8.3 \mathrm{~Hz}, 1 \mathrm{H}), 3.74(\mathrm{~d}, J=6.9 \mathrm{~Hz}$, $1 \mathrm{H}), 3.61(\mathrm{~s}, 6 \mathrm{H}), 3.44(\mathrm{~d}, J=4.6 \mathrm{~Hz}, 1 \mathrm{H}), 2.54 \sim 2.42(\mathrm{~m}$, $1 \mathrm{H}), 2.39(\mathrm{~d}, J=3.7 \mathrm{~Hz}, 1 \mathrm{H}), 2.35(\mathrm{~s}, 3 \mathrm{H}), 2.32 \sim 2.57(\mathrm{~m}$, $1 \mathrm{H}), 2.17(\mathrm{~s}, 3 \mathrm{H}), 1.81(\mathrm{t}, J=13.2 \mathrm{~Hz}, 1 \mathrm{H}), 1.73(\mathrm{~s}, 3 \mathrm{H})$, $1.64(\mathrm{~s}, 1 \mathrm{H}), 1.62(\mathrm{~s}, 3 \mathrm{H}), 1.17(\mathrm{~s}, 3 \mathrm{H}), 1.07(\mathrm{~s}, 3 \mathrm{H}) ;{ }^{13} \mathrm{C}$ NMR $\left(101 \mathrm{MHz}, \mathrm{CDCl}_{3}\right) \delta: 202.6,171.7,170.3,169.4$, $166.0,165.8,159.9,141.0,136.8,134.8,132.8,132.1$, 129.3, 128.0, 127.8, 127.3, 125.9, 104.0, 102.7, 83.4, 80.1, $78.2,74.5,73.9,72.2,71.3,71.2,57.6,54.4,53.8,44.6$, 42.1, 34.6, 25.8, 21.7, 20.9, 19.9, 13.8, 8.6. HRMS (ESI) calcd for $\mathrm{C}_{49} \mathrm{H}_{56} \mathrm{NO}_{16}[\mathrm{M}+\mathrm{H}]^{+}:$914.3599, found 914.3552.

3'-( $N$-呋喃甲酰基)紫杉醇 (14j): 白色固体 $17 \mathrm{mg}$, 产率 49\%. m.p. $166 \sim 168{ }^{\circ} \mathrm{C} ;{ }^{1} \mathrm{H}$ NMR $(400 \mathrm{MHz}$, $\left.\mathrm{CDCl}_{3}\right) \delta: 8.13(\mathrm{~d}, J=7.5 \mathrm{~Hz}, 2 \mathrm{H}), 7.63(\mathrm{t}, J=7.4 \mathrm{~Hz}, 1 \mathrm{H})$, $7.57 \sim 7.38(\mathrm{~m}, 7 \mathrm{H}), 7.35(\mathrm{t}, J=7.2 \mathrm{~Hz}, 1 \mathrm{H}), 7.16(\mathrm{~d}, J=$ $9.1 \mathrm{~Hz}, 1 \mathrm{H}), 7.02(\mathrm{~d}, J=3.5 \mathrm{~Hz}, 1 \mathrm{H}), 6.47$ (dd, $J=3.4,1.7$ $\mathrm{Hz}, 1 \mathrm{H}), 6.27$ (s, 1H), 6.24 (t, $J=9.2 \mathrm{~Hz}, 1 \mathrm{H}), 5.74$ (dd,
$J=9.1,2.1 \mathrm{~Hz}, 1 \mathrm{H}), 5.67(\mathrm{~d}, J=7.0 \mathrm{~Hz}, 1 \mathrm{H}), 4.94(\mathrm{~d}, J=$ $8.4 \mathrm{~Hz}, 1 \mathrm{H}), 4.77(\mathrm{dd}, J=5.2,2.6 \mathrm{~Hz}, 1 \mathrm{H}), 4.40 \sim 4.36(\mathrm{~m}$, $1 \mathrm{H}), 4.30(\mathrm{~d}, J=8.5 \mathrm{~Hz}, 1 \mathrm{H}), 4.20(\mathrm{~d}, J=8.5 \mathrm{~Hz}, 1 \mathrm{H}), 3.80$ (d, $J=6.9 \mathrm{~Hz}, 1 \mathrm{H}), 3.61(\mathrm{~d}, J=5.3 \mathrm{~Hz}, 1 \mathrm{H}), 2.60 \sim 2.50$ (m, 1H), $2.48(\mathrm{~d}, J=4.0 \mathrm{~Hz}, 1 \mathrm{H}), 2.37(\mathrm{~s}, 3 \mathrm{H}), 2.36 \sim 2.25$ (m, $2 \mathrm{H}), 2.24(\mathrm{~s}, 3 \mathrm{H}), 1.87 \sim 1.84(\mathrm{~m}, 1 \mathrm{H}), 1.81(\mathrm{~s}, 3 \mathrm{H})$, $1.69(\mathrm{~s}, 3 \mathrm{H}), 1.24(\mathrm{~s}, 3 \mathrm{H}), 1.15(\mathrm{~s}, 3 \mathrm{H}) ;{ }^{13} \mathrm{C}$ NMR $(101$ $\left.\mathrm{MHz}, \mathrm{CDCl}_{3}\right) \delta: 203.6,172.7,171.3,170.4,167.1,167.0$, 142.0, 138.0, 133.7, 133.6, 133.2, 132.0, 130.2, 129.2, 129.0, 128.8, 128.7, 128.4, 127.1, 127.0, 84.4, 81.2, 79.0, 76.5, 75.6, 74.9, 73.2, 72.3, 72.2, 58.6, 55.1, 45.7, 43.2, 35.7, 35.6, 26.9, 22.6, 21.8, 20.9, 14.8, 9.6. HRMS (ESI) calcd for $\mathrm{C}_{45} \mathrm{H}_{50} \mathrm{NO}_{15}[\mathrm{M}+\mathrm{H}]^{+}:$844.3180, found 844.3167.

3'-( $N$-噻吩甲酰基)紫杉醇(14k): 白色固体 $26 \mathrm{mg}$, 产率 59\%. m.p. 135 136 ${ }^{\circ} \mathrm{C} ;{ }^{1} \mathrm{H}$ NMR $(400 \mathrm{MHz}$, $\left.\mathrm{CDCl}_{3}\right) \delta: 8.12(\mathrm{~d}, J=7.3 \mathrm{~Hz}, 2 \mathrm{H}), 7.62(\mathrm{t}, J=7.4 \mathrm{~Hz}, 1 \mathrm{H})$, $7.55 \sim 7.49(\mathrm{~m}, 3 \mathrm{H}), 7.49 \sim 7.43(\mathrm{~m}, 3 \mathrm{H}), 7.41(\mathrm{t}, J=7.4$ $\mathrm{Hz}, 2 \mathrm{H}), 7.34$ (t, $J=7.1 \mathrm{~Hz}, 1 \mathrm{H}), 7.04(\mathrm{dd}, J=4.9,3.8 \mathrm{~Hz}$, $1 \mathrm{H}), 6.93(\mathrm{~d}, J=9.0 \mathrm{~Hz}, 1 \mathrm{H}), 6.27(\mathrm{~s}, 1 \mathrm{H}), 6.23$ (t, $J=9.1$ $\mathrm{Hz}, 1 \mathrm{H}), 5.75$ (dd, $J=8.9,2.4 \mathrm{~Hz}, 1 \mathrm{H}), 5.67(\mathrm{~d}, J=7.0 \mathrm{~Hz}$, $1 \mathrm{H}), 4.93(\mathrm{~d}, J=8.3 \mathrm{~Hz}, 1 \mathrm{H}), 4.78(\mathrm{~d}, J=2.2 \mathrm{~Hz}, 1 \mathrm{H}), 4.39$ (dd, $J=10.5,6.8 \mathrm{~Hz}, 1 \mathrm{H}), 4.28(\mathrm{~d}, J=8.4 \mathrm{~Hz}, 1 \mathrm{H}), 4.20$ $(\mathrm{d}, J=8.4 \mathrm{~Hz}, 1 \mathrm{H}), 3.88 \sim 3.60(\mathrm{~m}, 2 \mathrm{H}), 2.60 \sim 2.50(\mathrm{~m}$, $2 \mathrm{H}), 2.36(\mathrm{~s}, 3 \mathrm{H}), 2.34 \sim 2.24(\mathrm{~m}, 2 \mathrm{H}), 2.23(\mathrm{~s}, 3 \mathrm{H}), 1.87 \sim$ $1.82(\mathrm{~m}, 1 \mathrm{H}), 1.80(\mathrm{~s}, 3 \mathrm{H}), 1.68(\mathrm{~s}, 3 \mathrm{H}), 1.23(\mathrm{~s}, 3 \mathrm{H}), 1.14$ (s, 3H); ${ }^{13} \mathrm{C}$ NMR $\left(101 \mathrm{MHz}, \mathrm{CDCl}_{3}\right) \delta: 203.6,172.6$, $171.3,170.5,167.0,161.5,141.9,137.9,133.7,133.2$, $130.8,130.2,129.2,129.0,128.8,128.7,128.4,127.8$, 127.0, 84.4, 81.2, 79.0, 76.5, 75.6, 74.9, 73.2, 72.3, 72.2, 58.6, 55.0, 45.7, 43.2, 35.7, 35.7, 29.7, 22.6, 21.8, 20.9, 14.9, 9.6. HRMS (ESI) calcd for $\mathrm{C}_{45} \mathrm{H}_{50} \mathrm{NO}_{14} \mathrm{~S}[\mathrm{M}+\mathrm{H}]^{+}$: 860.2952 , found 860.2914 .

$3^{\prime}-(N$-对三氟甲基苯磺酰基)紫杉醇 (14m): 白色固 体 $14 \mathrm{mg}$, 产率 39\%. m.p. 186 188 ${ }^{\circ} \mathrm{C} ;{ }^{1} \mathrm{H}$ NMR (400 $\left.\mathrm{MHz}, \mathrm{CDCl}_{3}\right) \delta: 8.01(\mathrm{~d}, J=7.5 \mathrm{~Hz}, 2 \mathrm{H}), 7.59$ (d, $J=8.1$ $\mathrm{Hz}, 2 \mathrm{H}), 7.53(\mathrm{t}, J=7.4 \mathrm{~Hz}, 1 \mathrm{H}), 7.42-7.36(\mathrm{~m}, 4 \mathrm{H})$, $7.12 \sim 7.00(\mathrm{~m}, 3 \mathrm{H}), 6.97(\mathrm{~d}, J=7.2 \mathrm{~Hz}, 2 \mathrm{H}), 6.23 \sim 6.13$ (m, 2H), 6.09 (d, $J=9.2 \mathrm{~Hz}, 1 \mathrm{H}), 5.59(\mathrm{~d}, J=7.0 \mathrm{~Hz}, 1 \mathrm{H})$, $4.97 \sim 4.79(\mathrm{~m}, 2 \mathrm{H}), 4.46(\mathrm{t}, J=4.0 \mathrm{~Hz}, 1 \mathrm{H}), 4.34 \sim 4.25$ (m, 1H), $4.21(\mathrm{~d}, J=8.6 \mathrm{~Hz}, 1 \mathrm{H}), 4.13(\mathrm{~d}, J=8.6 \mathrm{~Hz}, 1 \mathrm{H})$, $3.76 \sim 3.61(\mathrm{~m}, 2 \mathrm{H}), 2.49(\mathrm{~d}, J=3.6 \mathrm{~Hz}, 1 \mathrm{H}), 2.53 \sim 2.38$ (m, 2H), $2.26(\mathrm{~s}, 3 \mathrm{H}), 2.17(\mathrm{~s}, 3 \mathrm{H}), 2.17 \sim 2.11(\mathrm{~m}, 2 \mathrm{H})$, $1.83 \sim 1.80(\mathrm{~m}, 1 \mathrm{H}), 1.73(\mathrm{~s}, 3 \mathrm{H}), 1.62(\mathrm{~s}, 3 \mathrm{H}), 1.17(\mathrm{~s}$, $3 \mathrm{H}), 1.07$ (s, 3H); ${ }^{13} \mathrm{C}$ NMR (101 MHz, $\left.\mathrm{CDCl}_{3}\right) \delta: 202.5$, 
$170.3,170.2,169.5,165.9,140.6,134.7,132.8,132.4$, $129.1,128.1,127.6,127.5,126.4,126.1,124.8$ (q, $J=3.6$ Hz), 83.3, 80.4, 78.0, 74.5, 73.9, 73.5, 71.3, 71.1, 58.8, 57.6, 44.6, 42.1, 34.6, 25.8, 21.6, 20.6, 19.8, 14.0, 8.6. HRMS (ESI) calcd for $\mathrm{C}_{47} \mathrm{H}_{51} \mathrm{~F}_{3} \mathrm{NO}_{15} \mathrm{~S}[\mathrm{M}+\mathrm{H}]^{+}$: 958.2932, found 958.2947.

13-[(4S,5R)-3-对三氟甲基苯磺酰基-2-(4-甲氧基苯 基)-4-苯基]-5-腎唑啉羧酸-巴卡汀 III 酯(cyclo-14m):白 色固体 $13 \mathrm{mg}$, 产率 34\%. m.p.143 $146{ }^{\circ} \mathrm{C} ;{ }^{1} \mathrm{H}$ NMR $\left(400 \mathrm{MHz}, \mathrm{CDCl}_{3}\right) \delta: 7.96(\mathrm{~d}, J=7.7 \mathrm{~Hz}, 2 \mathrm{H}), 7.54$ (t, $J=$ $7.3 \mathrm{~Hz}, 1 \mathrm{H}), 7.45(\mathrm{~d}, J=8.3 \mathrm{~Hz}, 2 \mathrm{H}), 7.44 \sim 7.23(\mathrm{~m}$, $11 \mathrm{H}), 6.79(\mathrm{~d}, J=8.4 \mathrm{~Hz}, 2 \mathrm{H}), 6.37(\mathrm{~s}, 1 \mathrm{H}), 6.22(\mathrm{~s}, 1 \mathrm{H})$, $6.18(\mathrm{t}, J=8.7 \mathrm{~Hz}, 1 \mathrm{H}), 5.58(\mathrm{~d}, J=7.0 \mathrm{~Hz}, 1 \mathrm{H}), 5.37(\mathrm{~d}$, $J=3.6 \mathrm{~Hz}, 1 \mathrm{H}), 4.82(\mathrm{~d}, J=9.1 \mathrm{~Hz}, 1 \mathrm{H}), 4.76(\mathrm{~d}, J=3.6$ $\mathrm{Hz}, 1 \mathrm{H}), 4.36$ (dd, $J=10.4,6.9 \mathrm{~Hz}, 1 \mathrm{H}), 4.18$ (d, $J=8.4$ $\mathrm{Hz}, 1 \mathrm{H}), 4.05$ (d, $J=8.4 \mathrm{~Hz}, 1 \mathrm{H}), 3.76$ (s, 3H), 3.71 (d, $J=$ $6.9 \mathrm{~Hz}, 1 \mathrm{H}), 2.52 \sim 2.41(\mathrm{~m}, 1 \mathrm{H}), 2.20(\mathrm{~s}, 3 \mathrm{H}), 2.17 \sim 2.07$ $(\mathrm{m}, 2 \mathrm{H}), 1.84(\mathrm{~s}, 3 \mathrm{H}), 1.78(\mathrm{~s}, 3 \mathrm{H}), 1.77 \sim 1.73(\mathrm{~m}, 1 \mathrm{H})$, $1.59(\mathrm{~s}, 3 \mathrm{H}), 1.21(\mathrm{~s}, 3 \mathrm{H}), 1.08(\mathrm{~s}, 3 \mathrm{H}) ;{ }^{13} \mathrm{C}$ NMR $(101$ $\left.\mathrm{MHz}, \mathrm{CDCl}_{3}\right) \delta: 202.6,170.3,169.0,168.3,166.0,159.8$, $140.9,140.7,137.3,132.8,132.3,129.0,128.4,128.1$, $128.0,127.8,127.6,127.1,126.4,126.2,124.7$ (q, $J=3.8$ Hz), 112.9, 92.3, 83.4, 81.4, 79.8, 78.3, 74.4, 73.9, 71.1, 70.9, 64.5, 57.5, 54.3, 44.5, 42.1, 34.6, 34.4, 28.7, 25.7, 20.9, 20.7, 19.8, 14.2, 8.5. HRMS (ESI) calcd for $\mathrm{C}_{55} \mathrm{H}_{56^{-}}$ $\mathrm{F}_{3} \mathrm{NO}_{16} \mathrm{SNa}[\mathrm{M}+\mathrm{Na}]^{+}: 1098.3170$, found 1098.3181 .

13-[(4S,5R)-3-对甲基苯磺酰基-2-(4-甲氧基苯基)-4苯基]-5-噁唑啉羧酸-巴卡汀 III 酯(cyclo-14n): 白色固体 $14 \mathrm{mg}$, 产率 36\%. m.p. $140 \sim 142{ }^{\circ} \mathrm{C} ;{ }^{1} \mathrm{H}$ NMR (400 $\left.\mathrm{MHz}, \mathrm{CDCl}_{3}\right) \delta: 8.03(\mathrm{~d}, J=7.4 \mathrm{~Hz}, 2 \mathrm{H}), 7.63$ (t, $J=7.4$ $\mathrm{Hz}, 1 \mathrm{H}), 7.57 \sim 7.44(\mathrm{~m}, 6 \mathrm{H}), 7.39 \sim 7.30(\mathrm{~m}, 5 \mathrm{H}), 7.21(\mathrm{~d}$, $J=8.0 \mathrm{~Hz}, 2 \mathrm{H}), 6.93(\mathrm{~d}, J=8.6 \mathrm{~Hz}, 2 \mathrm{H}), 6.45(\mathrm{~s}, 1 \mathrm{H}), 6.28$ (s, $1 \mathrm{H}), 6.10(\mathrm{t}, J=8.7 \mathrm{~Hz}, 1 \mathrm{H}), 5.64(\mathrm{~d}, J=7.0 \mathrm{~Hz}, 1 \mathrm{H})$, $5.25(\mathrm{~d}, J=4.7 \mathrm{~Hz}, 1 \mathrm{H}), 4.88(\mathrm{~d}, J=8.8 \mathrm{~Hz}, 1 \mathrm{H}), 4.71(\mathrm{~d}$, $J=4.7 \mathrm{~Hz}, 1 \mathrm{H}), 4.47 \sim 4.36(\mathrm{~m}, 1 \mathrm{H}), 4.24(\mathrm{~d}, J=8.4 \mathrm{~Hz}$, $1 \mathrm{H}), 4.11(\mathrm{~d}, J=8.4 \mathrm{~Hz}, 1 \mathrm{H}), 3.85$ (s, 3H), 3.74 (d, $J=7.0$ $\mathrm{Hz}, 1 \mathrm{H}), 2.57 \sim 2.48(\mathrm{~m}, 1 \mathrm{H}), 2.46(\mathrm{~d}, J=4.0 \mathrm{~Hz}, 1 \mathrm{H})$, $2.37(\mathrm{~s}, 3 \mathrm{H}), 2.25(\mathrm{~s}, 3 \mathrm{H}), 2.23 \sim 2.07(\mathrm{~m}, 2 \mathrm{H}), 1.86(\mathrm{~s}$, $3 \mathrm{H}), 1.78(\mathrm{~s}, 3 \mathrm{H}), 1.72(\mathrm{~s}, 1 \mathrm{H}), 1.65(\mathrm{~s}, 3 \mathrm{H}), 1.26(\mathrm{~s}, 3 \mathrm{H})$, 1.15 (s, 3H); ${ }^{13} \mathrm{C}$ NMR (101 MHz, $\left.\mathrm{CDCl}_{3}\right) \delta: 203.6,171.3$, $170.0,169.2,167.0,160.5,144.4,142.1,138.5,134.5$, $133.9,133.1,130.1,129.7,129.1,128.9,128.9,128.8$, $128.7,128.5,128.1,127.3,113.9,93.3,84.4,82.3,80.8$, $79.4,75.5,75.0,72.2,71.8,58.5,55.4,45.6,43.2,35.6$, 29.7, 26.8, 21.9, 21.7, 21.6, 20.9, 15.1, 9.5. HRMS (ESI) calcd for $\mathrm{C}_{55} \mathrm{H}_{60} \mathrm{NO}_{16} \mathrm{~S}[\mathrm{M}+\mathrm{H}]^{+}:$1022.3633, found 1022.3620 .

3.2.813-( $N$ - 苯甲酰基)-苯基异丝氨酸-7,10-二甲氧 基-10-去乙酰基巴卡汀 III 酯(15a)的合成

将化合物 $\mathbf{1 1}(0.1 \mathrm{mmol}) 、$ 化合物 $\mathbf{9 a}(0.23 \mathrm{mmol})$ 、 二甲氨基吡啶(DMAP, $0.23 \mathrm{mmol}$ )和 $2.5 \mathrm{~mL}$ 无水甲苯加 入 $50 \mathrm{~mL}$ 烧瓶中, 加热至 $70{ }^{\circ} \mathrm{C}$ 使反应液呈悬浊液, 之 后加入 DCC $(0.17 \mathrm{mmol})$, 反应 $15 \mathrm{~min}$ 后再加入 $\mathrm{DCC}$ $(0.16 \mathrm{mmol})$, 继续摚拌 $1 \mathrm{~h}$. TCL 和 LC-MS 监测反应, 反 应结束后过滤, 收集滤液. 最后以 $V$ (石油醚) : $V$ (乙酸 乙酯) $=1: 1$ 为洗脱剂柱层析分离得到白色固体 13a, 收率为 $92 \%$. 向所得白色固体 13a 中 $(0.1 \mathrm{mmol})$ 中加入 3 $\mathrm{mL}$ 无水乙醇, 呈白色浊液, 再向反应液中加入 $20 \%$ $30 \%$ 的 $\mathrm{HCl} / \mathrm{EtOH}$ 溶液 $0.5 \mathrm{~mL}$, 室温下搅拌 $24 \mathrm{~h}$, 反应液 澄清. LC-MS 监测反应, 反应结束后加入 $1.5 \mathrm{~mL}$ 饱和 $\mathrm{NaHCO}_{3}$ 溶液、 $0.5 \mathrm{~mL}$ 水和 $10 \mathrm{~mL}$ 乙酸乙酯萃取分液, 水相再用 $10 \mathrm{~mL}$ 乙酸乙酯反萃, 合并乙酸乙酯相, 干燥 浓缩. 最后以 $V$ (二氯甲烷) $: V$ (甲醇 $)=20: 1$ 为洗脱剂 柱层析分离得到 $42 \mathrm{mg}$ 白色固体 15a, 收率为 55\%. m.p. $221 \sim 222{ }^{\circ} \mathrm{C} ;{ }^{1} \mathrm{H}$ NMR $\left(400 \mathrm{MHz}, \mathrm{CDCl}_{3}\right) \delta: 8.03(\mathrm{~d}, J=$ $7.7 \mathrm{~Hz}, 2 \mathrm{H}), 7.71(\mathrm{~d}, J=7.7 \mathrm{~Hz}, 2 \mathrm{H}), 7.53(\mathrm{t}, J=7.2 \mathrm{~Hz}$, 1H), $7.49 \sim 7.38$ (m, 5H), $7.37 \sim 7.31(\mathrm{~m}, 4 \mathrm{H}), 7.27$ (t, $J=$ $7.2 \mathrm{~Hz}, 1 \mathrm{H}), 7.06(\mathrm{~d}, J=8.7 \mathrm{~Hz}, 1 \mathrm{H}), 6.12(\mathrm{t}, J=8.7 \mathrm{~Hz}$, $1 \mathrm{H}), 5.72(\mathrm{~d}, J=8.8 \mathrm{~Hz}, 1 \mathrm{H}), 5.56(\mathrm{~d}, J=6.8 \mathrm{~Hz}, 1 \mathrm{H}), 4.90$ (d, $J=9.4 \mathrm{~Hz}, 1 \mathrm{H}), 4.74 \sim 4.70(\mathrm{~m}, 1 \mathrm{H}), 4.69(\mathrm{~s}, 1 \mathrm{H}), 4.22$ (d, $J=8.4 \mathrm{~Hz}, 1 \mathrm{H}), 4.11(\mathrm{~d}, J=8.4 \mathrm{~Hz}, 1 \mathrm{H}), 3.82 \sim 3.68$ (m, 2H), $3.58(\mathrm{~d}, J=4.8 \mathrm{~Hz}, 1 \mathrm{H}), 3.36(\mathrm{~s}, 3 \mathrm{H}), 3.22(\mathrm{~s}$, $3 \mathrm{H}), 2.67 \sim 2.57(\mathrm{~m}, 1 \mathrm{H}), 2.30(\mathrm{~s}, 3 \mathrm{H}), 2.26 \sim 2.15(\mathrm{~m}$, $2 \mathrm{H}), 1.78 \sim 1.71(\mathrm{~m}, 1 \mathrm{H}), 1.70(\mathrm{~s}, 3 \mathrm{H}), 1.65(\mathrm{~s}, 3 \mathrm{H}), 1.60$ (s, 1H), $1.12(\mathrm{~s}, 3 \mathrm{H}) \cdot{ }^{13} \mathrm{C}$ NMR (101 MHz, $\left.\mathrm{CDCl}_{3}\right) \delta$ : 203.9, 171.4, 169.6, 165.9, 137.3, 137.0, 134.9, 132.7, $132.6,130.9,129.1,128.2,128.0,127.7,127.3,126.1$, $126.1,99.0,83.1,81.6,80.8,79.8,77.7,73.5,72.3,71.5$, 59.4, 56.4, 56.0, 55.9, 54.0, 46.4, 42.2, 34.4, 31.1, 30.9, 28.7, 25.9, 21.7, 19.6, 13.6, 13.2, 13.1, 9.3. HRMS (ESI) calcd for $\mathrm{C}_{47} \mathrm{H}_{54} \mathrm{NO}_{13}[\mathrm{M}+\mathrm{H}]^{+}$: 840.3595 , found 840.3544 .

13-( $N$-对二甲氨基苯甲酰基)苯基异丝氨酸-7,10-二 甲氧基-10-去乙酰基巴卡汀 III 酯(15h): 白色固体 19 mg, 产率 56\%. m.p. 232 234 ${ }^{\circ} \mathrm{C} ;{ }^{1} \mathrm{H}$ NMR $(400 \mathrm{MHz}$, $\left.\mathrm{CDCl}_{3}\right) \delta: 8.03(\mathrm{~d}, J=7.7 \mathrm{~Hz}, 2 \mathrm{H}), 7.61(\mathrm{~d}, J=8.7 \mathrm{~Hz}$, $2 \mathrm{H}), 7.54(\mathrm{t}, J=7.3 \mathrm{~Hz}, 1 \mathrm{H}), 7.46 \sim 7.36(\mathrm{~m}, 4 \mathrm{H}), 7.32(\mathrm{t}$, $J=7.3 \mathrm{~Hz}, 2 \mathrm{H}), 7.24(\mathrm{t}, J=7.9 \mathrm{~Hz}, 1 \mathrm{H}), 6.91(\mathrm{~d}, J=8.7$ $\mathrm{Hz}, 1 \mathrm{H}), 6.55$ (d, $J=8.6 \mathrm{~Hz}, 2 \mathrm{H}), 6.09$ (t, $J=8.6 \mathrm{~Hz}, 1 \mathrm{H})$, $5.68(\mathrm{~d}, J=7.9 \mathrm{~Hz}, 1 \mathrm{H}), 5.55(\mathrm{~d}, J=6.9 \mathrm{~Hz}, 1 \mathrm{H}), 4.90(\mathrm{~d}$, 
$J=9.2 \mathrm{~Hz}, 1 \mathrm{H}), 4.75 \sim 4.64(\mathrm{~m}, 2 \mathrm{H}), 4.22(\mathrm{~d}, J=8.3 \mathrm{~Hz}$, $1 \mathrm{H}), 4.10(\mathrm{~d}, J=8.3 \mathrm{~Hz}, 1 \mathrm{H}), 3.81 \sim 3.66(\mathrm{~m}, 2 \mathrm{H}), 3.35$ (s, $3 \mathrm{H}), 3.21(\mathrm{~s}, 3 \mathrm{H}), 2.93(\mathrm{~s}, 6 \mathrm{H}), 2.67 \sim 2.55(\mathrm{~m}, 1 \mathrm{H}), 2.31$ $(\mathrm{s}, 3 \mathrm{H}), 2.23 \sim 2.10(\mathrm{~m}, 2 \mathrm{H}), 1.80 \sim 1.73(\mathrm{~m}, 1 \mathrm{H}), 1.71(\mathrm{~s}$, $3 \mathrm{H}), 1.68(\mathrm{~s}, 1 \mathrm{H}), 1.64(\mathrm{~s}, 3 \mathrm{H}), 1.11(\mathrm{~s}, 6 \mathrm{H}) ;{ }^{13} \mathrm{C}$ NMR (101 $\left.\mathrm{MHz}, \mathrm{CDCl}_{3}\right) \delta: 205.0,172.6,170.6,167.1,166.9,152.7$, $138.7,138.4,135.6,133.7,130.2,129.2,128.9,128.7$, $128.1,127.5,120.1,111.0,84.1,82.6,81.7,80.8,78.7$, $77.4,77.1,76.7,76.6,74.5,73.8,72.3,57.3,57.1,56.9$, 55.2, 47.4, 43.2, 40.1, 35.5, 32.1, 26.9, 22.7, 20.7, 14.6, 10.4. HRMS (ESI) calcd for $\mathrm{C}_{49} \mathrm{H}_{59} \mathrm{~N}_{2} \mathrm{O}_{13}[\mathrm{M}+\mathrm{H}]^{+}$: 883.4017, found 883.4048 .

13-[N-(2-呋喃甲酰基)]-苯基异丝氨酸-7,10-二甲氧 基-10-去乙酰基巴卡汀 III 酯(15j): 白色固体 $20 \mathrm{mg}$, 产 率 54\%. m.p. 235 236 ${ }^{\circ} \mathrm{C}$; ${ }^{1} \mathrm{H}$ NMR (400 $\mathrm{MHz}, \mathrm{CDCl}_{3}$ ) $\delta: 8.03(\mathrm{~d}, J=7.5 \mathrm{~Hz}, 2 \mathrm{H}), 7.54(\mathrm{t}, J=7.4 \mathrm{~Hz}, 1 \mathrm{H}), 7.48 \sim$ $7.37(\mathrm{~m}, 5 \mathrm{H}), 7.33(\mathrm{t}, J=7.4 \mathrm{~Hz}, 2 \mathrm{H}), 7.26(\mathrm{t}, J=7.2 \mathrm{~Hz}$, $1 \mathrm{H}), 7.18$ (d, $J=3.5 \mathrm{~Hz}, 1 \mathrm{H}), 6.99$ (d, $J=3.3 \mathrm{~Hz}, 1 \mathrm{H}), 6.41$ $(\mathrm{dd}, J=3.2,1.5 \mathrm{~Hz}, 1 \mathrm{H}), 6.13(\mathrm{t}, J=8.5 \mathrm{~Hz}, 1 \mathrm{H}), 5.68(\mathrm{dd}$, $J=9.1,2.2 \mathrm{~Hz}, 1 \mathrm{H}), 5.55(\mathrm{~d}, J=6.9 \mathrm{~Hz}, 1 \mathrm{H}), 4.89$ (d, $J=$ $9.0 \mathrm{~Hz}, 1 \mathrm{H}), 4.73 \sim 4.65(\mathrm{~m}, 2 \mathrm{H}), 4.21(\mathrm{~d}, J=8.4 \mathrm{~Hz}, 1 \mathrm{H})$, $4.12(\mathrm{~d}, J=8.4 \mathrm{~Hz}, 1 \mathrm{H}), 3.81 \sim 3.70(\mathrm{~m}, 2 \mathrm{H}), 3.62(\mathrm{~d}, J=$ $4.9 \mathrm{~Hz}, 1 \mathrm{H}), 3.36(\mathrm{~s}, 3 \mathrm{H}), 3.22(\mathrm{~s}, 3 \mathrm{H}), 2.67 \sim 2.56(\mathrm{~m}$, $1 \mathrm{H}), 2.28(\mathrm{~s}, 3 \mathrm{H}), 2.22 \sim 2.15(\mathrm{~m}, 2 \mathrm{H}), 1.80 \sim 1.74(\mathrm{~m}$, $1 \mathrm{H}), 1.73(\mathrm{~s}, 3 \mathrm{H}), 1.65(\mathrm{~s}, 3 \mathrm{H}), 1.61(\mathrm{~s}, 1 \mathrm{H}), 1.12(\mathrm{~s}, 6 \mathrm{H})$; ${ }^{13} \mathrm{C}$ NMR (101 MHz, $\mathrm{CDCl}_{3}$ ) $\delta: 204.8,172.1,170.6,166.9$, $157.8,147.4,144.2,138.3,137.9,135.9,133.6,130.2$, $129.3,128.9,128.6,128.3,127.2,115.1,112.3,84.1,82.7$, $81.9,80.8,78.7,77.3,77.0,76.7,74.5,73.5,72.4,57.4$, 57.0, 54.3, 47.5, 43.3, 35.4, 32.2, 27.0, 22.7, 20.6, 14.6, 10.3. HRMS (ESI) calcd for $\mathrm{C}_{45} \mathrm{H}_{51} \mathrm{NO}_{14} \mathrm{Na}[\mathrm{M}+\mathrm{Na}]^{+}$: 852.3207, found 852.3229.

13-[N-(2-噻吩甲酰基)]-苯基异丝氨酸-7,10-二甲氧 基-10-去乙酰基巴卡汀 III 酯(15k): 白色固体 $23 \mathrm{mg}$, 产 率 57\%. m.p. 230 231 ${ }^{\circ} \mathrm{C}$; ${ }^{1} \mathrm{H}$ NMR (400 $\mathrm{MHz}, \mathrm{CDCl}_{3}$ ) $\delta: 8.03(\mathrm{~d}, J=7.7 \mathrm{~Hz}, 2 \mathrm{H}), 7.53(\mathrm{t}, J=7.3 \mathrm{~Hz}, 1 \mathrm{H}), 7.49 \sim$ $7.37(\mathrm{~m}, 6 \mathrm{H}), 7.33(\mathrm{t}, J=7.3 \mathrm{~Hz}, 2 \mathrm{H}), 7.27(\mathrm{t}, J=7.3 \mathrm{~Hz}$, $1 \mathrm{H}), 6.99$ (t, $J=4.2 \mathrm{~Hz}, 1 \mathrm{H}), 6.90(\mathrm{~d}, J=8.9 \mathrm{~Hz}, 1 \mathrm{H}), 6.13$ $(\mathrm{t}, J=8.6 \mathrm{~Hz}, 1 \mathrm{H}), 5.69$ (d, $J=8.9 \mathrm{~Hz}, 1 \mathrm{H}), 5.55(\mathrm{~d}, J=6.8$ $\mathrm{Hz}, 1 \mathrm{H}), 4.89(\mathrm{~d}, J=9.3 \mathrm{~Hz}, 1 \mathrm{H}), 4.75 \sim 4.66(\mathrm{~m}, 2 \mathrm{H})$, $4.21(\mathrm{~d}, J=8.3 \mathrm{~Hz}, 1 \mathrm{H}), 4.12(\mathrm{~d}, J=8.3 \mathrm{~Hz}, 1 \mathrm{H}), 3.81 \sim$ 3.69 (m, 2H), 3.61 (d, $J=4.4 \mathrm{~Hz}, 1 \mathrm{H}), 3.36$ (s, 3H), 3.22 $(\mathrm{s}, 3 \mathrm{H}), 2.67 \sim 2.56(\mathrm{~m}, 1 \mathrm{H}), 2.28(\mathrm{~s}, 3 \mathrm{H}), 2.24 \sim 2.13(\mathrm{~m}$, $2 \mathrm{H}), 1.79 \sim 1.72(\mathrm{~m}, 1 \mathrm{H}), 1.71(\mathrm{~s}, 3 \mathrm{H}), 1.65(\mathrm{~s}, 3 \mathrm{H}), 1.60$ $(\mathrm{s}, 1 \mathrm{H}), 1.12(\mathrm{~s}, 6 \mathrm{H}) ;{ }^{13} \mathrm{C} \mathrm{NMR}\left(101 \mathrm{MHz}, \mathrm{CDCl}_{3}\right) \delta$ :
$203.8,171.2,169.7,165.94,160.3,137.2,137.1,136.9$, $135.0,132.6,129.6,129.1,128.3,127.9,127.7,127.6$, 127.3, 126.7, 126.1, 83.1, 81.7, 80.9, 79.8, 77.7, 76.3, 76.0, $75.7,73.5,72.4,71.5,56.4,56.0,53.9,46.5,42.3,34.5$, $31.2,26.0,21.7,19.6,13.6,9.3$. HRMS (ESI) calcd for $\mathrm{C}_{45} \mathrm{H}_{51} \mathrm{NO}_{13} \mathrm{SNa}[\mathrm{M}+\mathrm{Na}]^{+}:$868.2979, found 868.2970.

辅助材料(Supporting Information) 化合物 7a $\sim 70$ 、化 合物 8a $\sim 80$ 、化合物 $14 \mathrm{~b}, 14 \mathrm{e} \sim 14 \mathrm{~m}$ 、化合物 $15 \mathrm{a} 、 15 \mathrm{~h}$ 、 $15 \mathbf{j} 、 15 \mathrm{k}$ 的核磁共振氢谱、碳谱谱图. 这些材料可以免 费从本刊网站(http://sioc-journal.cn/)上下载.

\section{References}

[1] (a) Yu, Y.; Wang, J. F.; Wang, C. Y.; Li, Y. X. Chin. J. Mod. Appl. Pharm. 2012, 29, 16 (in Chinese).

(于跃, 王军飞, 王长云, 李英霞, 中国现代应用药学, 2012, 29, 16.)

(b) Sheng, J. J. M.S. Thesis, East China Normal University, Shanghai, 2018 (in Chinese). (盛家骏, 硕士论文, 华东师范大学, 上海, 2018.)

[2] Rowinsky, E. K. Annu. Rev. Med. 1997, 48, 353-374.

[3] Shi, Q. W. Chin. Tradit. Herb. Drugs 2011, 42, 1878 (in Chinese). (史清文，中草药, 2011, 42, 1878.)

[4] (a) Nicolaou, K. C.; Yang, Z.; Liu, J. J.; Ueno, H.; Nantermet, P. G.; Guy, R. K.; Claiborne, C. F.; Renaud, J.; Couladouros, E. A.; Paulvannan, K.; Sorensen, E. J. Nature 1994, 367, 630.

(b) Nicolaou, K. C.; Dai, W. M.; Guy, R. K. Angew. Chem., Int. Ed. 1994, 33, 15.

(c) Holton, R. A.; Somoza, C.; Kim, H. B.; Liang, F.; Biediger, R. J.; Boatman, P. D.; Shindo, M.; Smith, C. C.; Kim, S.; Nadizadeh, H.; Suzuki, Y.; Tao, C.; Vu, P.; Tang, S.; Zhang, P.; Murthi, K. K.; Gentile, L. N.; Liu, J. H. J. Am. Chem. Soc. 1994, 116, 1597.

(d) Danishefsky, S. J.; Masters, J. J.; Young, W. B.; Link, J. T.; Snyder, L. B.; Magee, T. V.; Jung, D. K.; Isaacs, R. C. A.; Bornmann, W. G.; Alaimo, C. A.; Coburn, C. A.; Grandi, M. J. D. J. Am. Chem. Soc. 1996, 118, 2843.

(e) Wender, P. A.; Badham, N. F.; Conway, S. P.; Floreancig, P. E.; Glass, T. E.; Houze, J. B.; Crauss, N. E.; Lee, D.; Marquess, D. G.; McGrane, P. L.; Meng, W.; Natchus, M. G.; Shuker, A. J.; Sutton, J. C.; Taylor, R. E. J. Am. Chem. Soc. 1997, 119, 2755.

(f) Morihira, K.; Hara, R.; Kawahara, S.; Nishimori, T.; Nakamura, N.; Kusama, H.; Kuwajima, I. J. Am. Chem. Soc. 1998, 120, 12980. (g) Mukaiyama, T.; Shiina, I.; Iwadare, H.; Saitoh, M.; Nishimura, T.; Ohkawa, N.; Sakoh, H.; Nishimura,K.; Tani, Y.; Hasegawa, M.; Yamada, K.; Saitoh, K. Chem.-Eur. J. 1999, 5, 121.

(h) Doi, T.; Fuse S.; Miyamoto, S.; Nakai, K.; Sasuga, D.; Takahashi, T. Chem.-Asian J. 2006, 1, 370.

[5] (a) Guenard, D.; Gueritte-Voegelein, F.; Potier, P. Acc. Chem Res. 1993, 26, 160.

(b) Ojima, I.; Habus, I.; Zhao, M.; Zucco, M.; Park, Y. H.; Sun, C. M.; Brigaud, T. Tetrahedron 1992, 34, 6985.

[6] (a) Denis, J. N.; Greene, A. E.; Serra, A. A.; Luche, M. J. J. Org. Chem. 1986,51, 46.

(b) Deng, L.; Jacobson, E. N. J. Org. Chem. 1992, 57, 4320.

(c) Li, G.; Chang, H. T.; Sharpless, K. B. Angew. Chem., Int. Ed. Engl. 1996, 35, 451.

[7] Sharpless, K. B.; Wang, Z. M.; Kolb, H. C. J. Org. Chem. 1994, 59, 5104.

[8] (a) Liu, W.; Lv, B.; Gong, L. Angew. Chem., Int. Ed. 2009, 48, 6503.

(b) Bergmeier, S. C. Tetrahedron 2000, 56, 2561.

(c) Larrow, J. F.; Schaus, S. E.; Jacobsen, E. N. J. Am. Chem. Soc. 
1996, 118, 7420 .

(d) Olofsson, B.; Somfai, P. J. Org. Chem. 2002, 67, 8574.

(e) Hu, X. E. Tetrahedron 2004, 60, 2701.

[9] (a) Qian, Y.; Xu, X. F.; Jiang, L. Q.; Prajapati, D.; Hu, W. H. J. Org. Chem. 2010, 75, 7483.

(b) Guo, Z. Q.; Shi, T. D.; Jiang, J.; Yang, L. P.; Hu, W. H. Org. Biomol. Chem. 2009, 7, 5028.

[10] (a) Torssell, S.; Kienle, M.; Somfai, P. Angew. Chem., Int. Ed. 2005, 44, 3096 . (b) Torssell, S.; Somfai, P. Adv. Synth. Catal. 2006, 348, 2421.

(c) Sheng, J. J.; Chang, H.; Qian, Y.; Ma, M. L.; Hu, W. H. Tetrahedron Lett. 2018, 59, 2141.

[11] Xu, X. F.; Guo, X.; Han, X. C.; Yang, L. P.; Hu, W. H. Org. Chem. Front. 2014, 1, 181.

[12] Verkade, J.; Van Hemert, L.; Quaedflieg, P.; Alsters, P.; Van Delft, F.; Rutjes, F. Tetrahedron Lett. 2006, 47, 8109.

Li, Q. F.; Lin, H. X.; Cui, Y. M.; Xu, P. P. Eur. J. Med. Chem. 2015, 104, 97.

(Li, L.) 